OPEN ACCESS

Edited by:

Patricia Sampedro-Piquero,

University of Malaga, Spain

Reviewed by:

Małgorzata Helena Lehner, Instytut Psychiatrii i Neurologii w

Warszawie (IPiN), Poland

Begega Losa Azucena, Universidad de Oviedo Mieres,

Spain

*Correspondence: Juan C. Brenes

brenesaenz@gmail.com; juan.brenessaenz@ucr.ac.cr

Specialty section: This article was submitted to

Neuropharmacology, a section of the journal

Frontiers in Pharmacology

Received: 01 March 2020 Accepted: 24 April 2020

Published: 12 May 2020

Citation:

Rojas-Carvajal M, Sequeira-Cordero A and Brenes JC (2020)

Neurobehavioral Effects of Restricted and Unpredictable Environmental

Enrichment in Rats.

Front. Pharmacol. 11:674. doi: 10.3389/fphar.2020.00674

\section{Neurobehavioral Effects of Restricted and Unpredictable Environmental Enrichment in Rats}

\author{
Mijail Rojas-Carvajal ${ }^{1,2}$, Andrey Sequeira-Cordero ${ }^{1,3}$ and Juan C. Brenes ${ }^{1,2^{*}}$ \\ ${ }^{1}$ Neuroscience Research Center, University of Costa Rica, San Pedro, Costa Rica, ${ }^{2}$ Institute for Psychological Research, \\ University of Costa Rica, San Pedro, Costa Rica, ${ }^{3}$ Institute for Health Research, University of Costa Rica, San Pedro, Costa Rica
}

To study how motivational factors modulate experience-dependent neurobehavioral plasticity, we modify a protocol of environmental enrichment (EE) in rats. We assumed that the benefits derived from EE might vary according to the level of incentive salience attributed to it. To enhance the rewarding properties of EE, access to the EE cage varied randomly from 2 to $48 \mathrm{~h}$ for 30 days (REE). The REE group was enriched only $50 \%$ of the time and was compared to standard housing and continuous EE (CEE) groups. As behavioral readout, we analyzed the spontaneous activity and the ultrasonic vocalizations (USVs) within the EE cage weekly, and in the open field test at the end of the experiment. In the cage, REE increased the utilization of materials, physical activity, and the rate of appetitive USVs. In the OF, the CEE-induced enhancements in novelty habituation and social signaling were equaled by the REE. At the neural level, we measured the expression of genes related to neural plasticity and epigenetic regulations in different brain regions. In the dorsal striatum and hippocampus, REE upregulated the expression of the brainderived neurotrophic factor, its tropomyosin kinase $B$ receptor, and the DNA methyltransferase 3A. Altogether, our results suggest that the higher activity within the cage and the augmented incentive motivation provoked by the REE boosted its neurobehavioral effects equaling or surpassing those observed in the CEE condition. As constant exposures to treatments or stimulating environments are virtually impossible for humans, restricted EE protocols would have greater translational value than traditional ones.

Keywords: environmental enrichment, neural plasticity, motivation, ultrasonic vocalizations, grooming, learning, emotion, reward

\section{INTRODUCTION}

Knowing about how animals react to different environmental conditions would contribute to explaining why environmental stimulation in humans (e.g., physical and psychological therapies, exercise, and preventive or palliative treatments) benefits some subjects but not others, a crucial enigma about the complex relationship between experience and neurobehavioral plasticity. The positive impact of environmental stimulation in humans may rely upon attributions and expectations about their own performance and the putative benefits derived from the treatments 
(Benedetti et al., 2004; Knutson et al., 2005; Scott et al., 2007), suggesting that motivational factors are key modulators of the entire effect. However, the study of how motivation contributes to the benefits of environmental stimulation has received little attention in health sciences, and even less in preclinical research.

To study the likely role of motivation in the regulation of experience-dependent plasticity on brain and behavior, we first redesigned a protocol of environmental enrichment $(\mathrm{EE})$ in rats and then assessed its effects on different behavioral domains and the expression of genes related with neural plasticity (Figure 1; Table 1). EE consists of exposing laboratory rodents to physical and social stimulation higher than the one received in standard laboratory housing (Rosenzweig and Bennett, 1996; Crofton et al., 2015), with physical exercise, social activity, and the
A

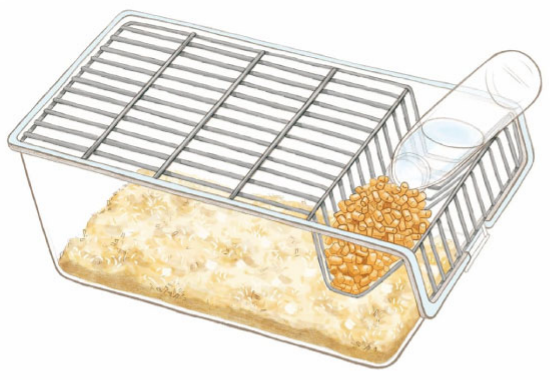

C

\begin{tabular}{ccccc}
\hline Group & Cage & Duration & $\begin{array}{c}\text { Physical } \\
\text { Context }\end{array}$ & $\begin{array}{c}\text { Social } \\
\text { Context }\end{array}$ \\
\hline SH2 & A & $720 \mathrm{~h}$ & Invariable & Simple \\
SH5 & A & $720 \mathrm{~h}$ & Invariable & Standard \\
REE & A & $360 \mathrm{~h}$ & Invariable & Increased \\
CEE & B & $360 \mathrm{~h}$ & Variable & \\
\hline
\end{tabular}

B

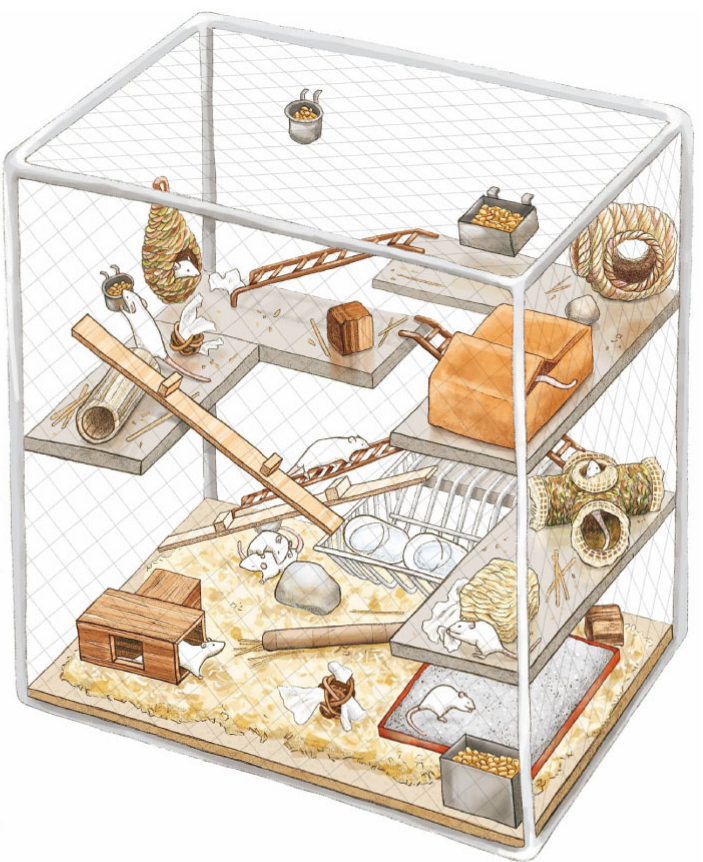

D

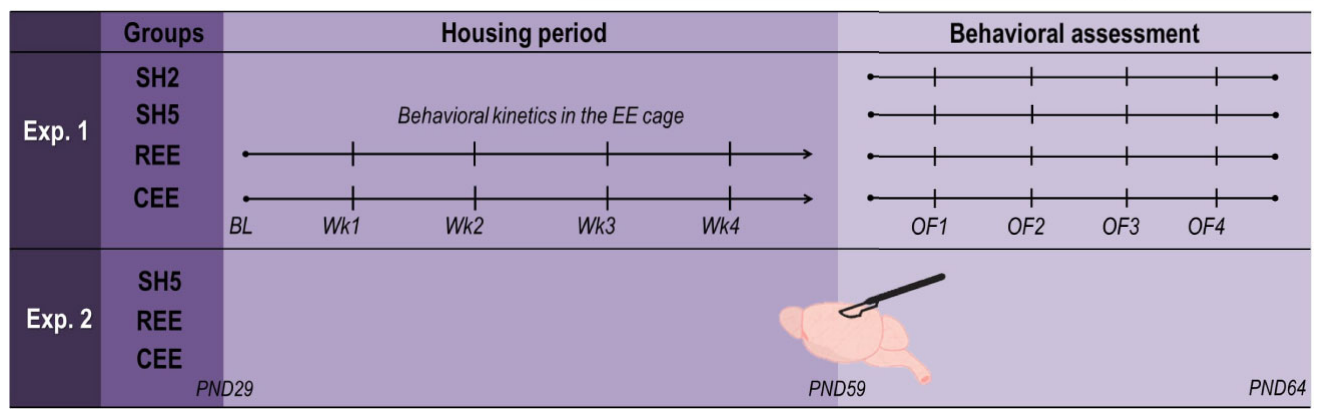

FIGURE 1 | Housing conditions and experimental design. (A) Transparent, polycarbonate cage $(56 \mathrm{~cm} \times 35 \mathrm{~cm} \times 20 \mathrm{~cm})$ filled with $\sim 5 \mathrm{~L}$ of bedding for standard housing (SH). (B) Custom-made $(135 \mathrm{~cm} \times 68 \mathrm{~cm} \times 110 \mathrm{~cm})$, environmental enrichment (EE) cage surrounded by wire-mesh walls consisting of four stainless steel levels interconnected by metal and wooden stairs. The cage contained dens, hideouts, nesting and chewing materials, different objects for sensorimotor stimulation, feeders, and two bottles of water (for details see Table 1). The first floor was always covered with bedding and had a sandbox. No running wheels were used. (C) Distribution of groups ( $\mathrm{n}=10$ rats per group). SH2: pair-housed rats in SH cages; SH5: five rats per cage in SH cages; CEE: ten rats per cage exposed continuously to EE throughout the 30-days protocol ( $720 \mathrm{~h}$ ), except during bed changes (30 min) when rats were housed in groups of five in SH cages; REE: 10 rats per cage with restricted and unpredictable access to the EE cage during $\sim 360 \mathrm{~h}(50 \%$ of the CEE total time). For the REE rats, the time in the cage was equally distributed between the light and dark cycle, with exposures to the EE cage ranging randomly from 2 to $48 \mathrm{~h}$. During the non-EE periods, these animals were also housed in groups of five in SH cages. CEE and REE rats were housed in independent EE cages. In order to control the increased handling experienced by REE rats, both SH groups and CEE rats were also handled each time REE rats were put in and out the EE cage: while $\mathrm{SH} 2$ and SH5 animals were relocated into new cages, CEE rats were group-housed in SH cages (fiveper cage) during 5 min and then returned into the EE cage. (D) Experimental design. Housing conditions started at postnatal (PND) 29 and continued throughout 30 days. In experiment 1, behavioral activity and USVs within the EE cage were measured once a week (WK) after bed changes. At PND 59, animals were tested on four, one-day apart open-field (OF) tests for $15 \mathrm{~min}$. In experiment 2, SH5, CEE, and REE groups were included ( $n=$ 10 rats per group) and animals were housed exactly as in experiment 1 . No behavioral assessments were carried out to these animals. At PND 59, rats were euthanized, and their brain tissues were collected for mRNA quantification of BDNF, TrkB, CREB, p250GAP, and DNMT3A genes on the hippocampus, the dorsal striatum, and the nucleus accumbens. 
TABLE 1 | A detailed description of the items used for environmental enrichment.

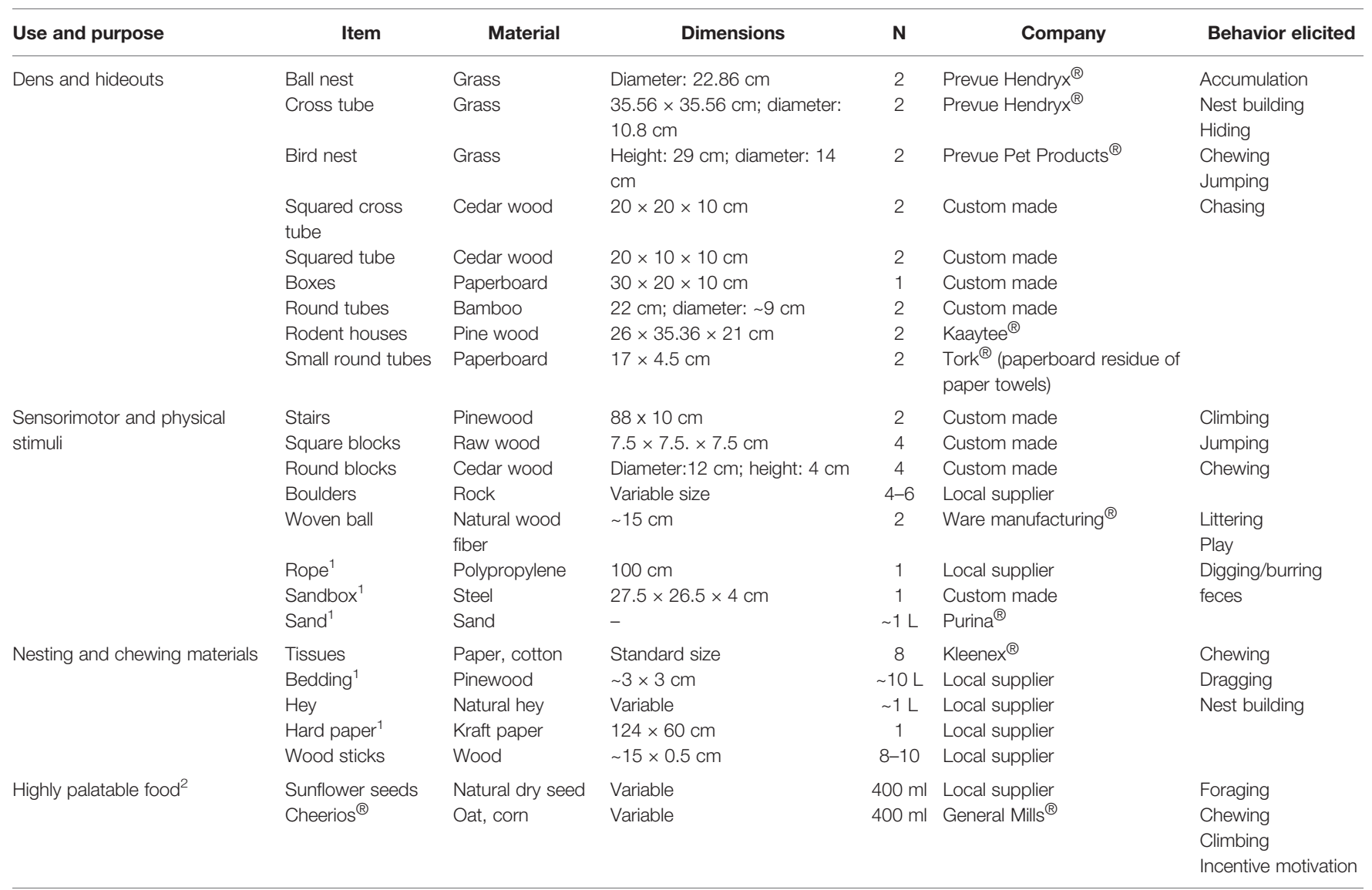

${ }^{1}$ These objects were always included in all the configurations of the cage.

exposure to complex and constantly changing stimuli as the triad of enriching factors affecting behavior and brain function (Bekinschtein et al., 2011; Crofton et al., 2015; Brenes et al., 2016; Ohline and Abraham, 2019). We assumed, therefore, that the benefits derived from EE may be determined by motivational factors related to how much animals attribute incentive salience to such stimulations. The motivational value of an appetitive stimulus can be potentiated through unpredictable presentations (Anselme et al., 2013; Schultz, 2016; Kreisler et al., 2018). Thus, to enhance the rewarding properties of the EE, we developed a protocol in which access to the EE cage was restricted to specific durations ranging from 2 to $48 \mathrm{~h}$ unpredictably for 30 days. Rats in the restricted and unpredictable EE (REE) condition were compared with rats exposed continuously to EE (CEE), with REE animals remaining only $50 \%$ of the total time in the EE cage throughout the housing period (Figures 1C, D). Contrary to most EE protocols described so far, our EE protocols consisted of a selection of natural, previously screened stimuli, which were classified into broad categories according to the function they may serve (e.g., dens and hideouts, sensorimotor and physical stimuli, nesting and chewing materials, and highly palatable foods). During the non-EE period, the REE rats were housed five in standard housing (SH) cages. Thus, two control groups were used: one with five rats per cage (SH5) to match the housing of REE rats, and a group with two rats per cage (SH2) with less social contact, which served as a control group of all other conditions.

Experiment 1 consisted of two phases. In the first phase, we evaluated the psychomotor and motivational responses toward the EE cage and compared the two EE protocols in that regard. Accordingly, we assessed once a week the exploratory activity, the use of materials, the eating-related behaviors, the social interactions, and the ultrasonic vocalizations (USVs) within the EE cages (Figure 4C). USVs are socio-affective signals serving distinct communicative functions in the rat. The so-called $22-\mathrm{kHz}$ calls are emitted as alarm signals in aversive situations, whereas $50-\mathrm{kHz}$ calls consist of many different subtypes appearing in social and non-social situations of neutral or positive affective valence (Brudzynski, 2013; Simola and Brudzynski, 2018). In the second phase, we analyzed the spontaneous activity and the USVs emitted in the open field (OF) after the 30-days housing period, to identify the behavioral effects of our EE protocols, which were compared between each other and with the $\mathrm{SH}$ groups. The $\mathrm{OF}$ was selected as it is one of the most consistent behavioral paradigms to detect the effects of EE (Elliott and Grunberg, 2005; Brenes Sáenz et al., 2006; Sampedro-Piquero et al., 2018). Besides measuring locomotion and rearing, we 
performed a detailed analysis of the different grooming subtypes as they may be informative of particular learning and emotional processes (e.g., novelty habituation and emotional de-arousal) and because they are very responsive to EE (Figure 2) (Spruijt et al., 1992; Brenes et al., 2009; Rojas-Carvajal et al., 2018). We also measured in the OF the spontaneous $50-\mathrm{kHz}$ calls elicited when rats are transiently separated from conspecifics (Figure 7, lower right panel) (Wöhr et al., 2008; Natusch and Schwarting, 2010). These USVs are considered as an index of prosocial behavior co-occurring during risk assessment, which can be modulated by social and physical EE and are responsive to repeated testing and stress (Schwarting et al., 2007; Wöhr et al., 2008; Brenes et al., 2016, Rojas-Carvajal and Brenes, submitted).

In experiment 2, we analyzed the effects of our EE protocols on the expression of genes involved in neural plasticity. We focused on genes related to the signaling pathway of the brainderived neurotrophic factor (BDNF) through its tropomyosin kinase $\mathrm{B}$ receptor (TrkB). Thus, we also measured the expression of the transcription factor cAMP response element-binding (CREB), which not only belongs to the BDNF/TrkB pathway but also regulates the expression of hundreds of genes, including BDNF (Carlezon et al., 2005; Yu and Rasenick, 2012). The expression of the Rho GTPase activating protein 32 (ARHGAP32, also known as p250GAP) was also analyzed as it is involved in structural plasticity by regulating the molecular changes associated with cytoskeleton remodeling (Nakazawa et al., 2003; Marler et al., 2014). Finally, we measured the expression of the DNA methyltransferase 3A (DNMT3A), as it is responsible for de novo-type DNA methylation and for establishing and maintaining proper DNA methylation patterns, which are presumably relevant for neural plasticity at certain genomic loci in postmitotic neurons (Griñan-Ferré et al., 2016; Zhang et al., 2018). These mRNA analyses were performed in SH5, REE, and CEE rats that were not submitted to behavioral assessment. We extracted the nucleus accumbens, dorsal striatum, and hippocampus because 1) they are all involved in different phases of the motivational processes (e.g., attribution of incentive salience, the transition from motivation to action, and contextual encoding of reward cues) (Williams and Undieh, 2010; Anselme et al., 2013; Schultz, 2016); 2) they play a pivotal role on learning and memory (e.g., associative, procedural, and episodic/spatial memory) (Richard et al., 2013; Lisman et al., 2017); and 3) the EE-induced physiological and cellular effects have been well identified in those regions and especially in hippocampus (Bezard et al., 2003; Brenes et al., 2008; Brenes and Fornaguera, 2008; Tipyasang et al., 2014; Brenes et al., 2016; Grimm et al., 2018; Scala et al., 2018; Ohline and Abraham, 2019).

\section{MATERIALS AND METHODS}

\section{Subjects}

Seventy male Wistar rats were transported to our colony room from LEBi facilities (University of Costa Rica, San José) at postnatal day (PND) 22 ( \pm 1 ). Upon arrival, animals were tail-marked, weighted, and housed with their littermates in a 12:12 light-dark schedule (lights on at 6:00 h), temperature of $22.3^{\circ} \mathrm{C}\left( \pm 4.5^{\circ} \mathrm{C}\right)$, and relative humidity of $71.5 \%$ with 10 air cycles/hour. Food and water were provided ad libitum and

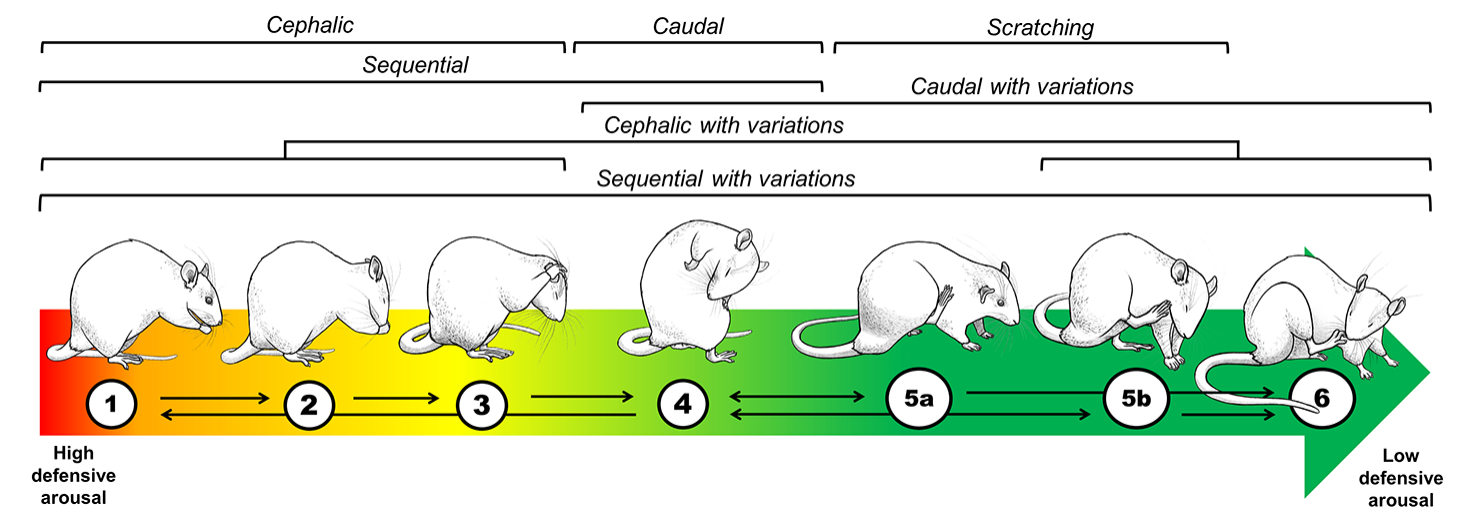

FIGURE 2 | Topographical and theoretical analysis of grooming. To understand why a non-exploratory, self-oriented behavior is displayed in the context of unconditioned anxiety tests, such as the OF, we developed a classification considering the kinetics, the anatomical distribution, and the complexity of grooming strokes (Brenes et al., 2009; Rojas-Carvajal et al., 2018; Rojas-Carvajal and Brenes, submitted). Based on its anatomical distribution, grooming was classified as cephalic (1-3), caudal (4), and sequential (1-4). Based on its motor complexity, each anatomical category could also include variations as follows: cephalic (1-3 + 5b, 6), caudal (4 + 5a, 6) and sequential (1-4 + 5a, $5 b, 6)$. Regarding the kinetics, the colored arrow represents the theoretical association between grooming subtypes and defensiveness over time. The red-to-green fading indicates the transition from high defensive arousal to low defensive arousal, with cephalic subtypes appearing at the beginning of testing when exploratory and riskassessment behaviors are prominent (Brenes et al., 2009; Rojas-Carvajal et al., 2018). With time, sequential grooming with variations gradually appears. As complex and intricate grooming sequences seem to compromise the prompt responses to any oncoming threat, it supposes that rats are now disengaged from displaying defensive responses. Thus, we interpreted the appearance of those subtypes as markers of habituation learning and emotional de-arousal (Brenes et al., 2009; Rojas-Carvajal et al., 2018). Black arrows represent the most common transition between the grooming sequences. 
refilled twice per week during bed changes. In experiment 1 , the 40 rats were screened in the cage test (CT) as previously reported (Pereira et al., 2014; Brenes et al., 2016). Briefly, the CT consisted of placing a rat individually in a transparent polycarbonate cage $(42 \times 26.5 \times 15 \mathrm{~cm})$ filled with fresh bedding and illuminated at $\sim 10$ lumens, where locomotion, rearing, total grooming, and total $50-\mathrm{kHz}$ USVs were measured for $5 \mathrm{~min}$ (see below for details). We left only one day of acclimatization between arrival and the CT because the experience of being moved from one cage to another and then returned back to the home cage constitutes a quite standard procedure in any lab, and because after the 5-min test rats remained undisturbed for four days, which can be considered a continuation of the acclimatization period. The groups' allocation procedure consisted of assigning semirandomly the rats to the experimental groups ( $n=10$ /group) based on body weight, litter of origin, behavioral activity, and USVs in the CT (Figures 1C, D), so that the inter-subject variability was equally distributed within the groups, and no subjects were excluded from the sample. In experiment 2 (30 rats), only body weight and the litter of origin were used for group allocation (Figure 1D). Housing conditions started at PND 29 in both experiments. All experimental procedures were done according to the guidelines of the Costa Rican Ministry of Science and Technology for the Care and Use of Laboratory Animals and were approved by the Institutional Committee for Animal Care and Use of the University of Costa Rica (CICUA161-16).

\section{Housing Conditions}

A modified version of our EE protocol was implemented (Brenes Sáenz et al., 2006; Brenes et al., 2009; Mora-Gallegos et al., 2015; Brenes et al., 2016; Rojas-Carvajal et al., 2018). To alleviate the stress of captivity, satisfy some of the ethological needs of the animals, and elicit species-specific behaviors, the proper stimuli should be provided (Olsson and Dahlborn, 2002; Swaisgood, 2007; Tarou and Bashaw, 2007). With this in mind, we adopted a rather naturalistic approach by replacing plastic objects with natural items to the largest extent possible (Figure 1B). Based on our previous protocol (Brenes et al., 2016) and pilot studies, we selected several items (see Table 1) which were supposed to serve a specific purpose classified into four broad categories: dens and hideouts, sensorimotor and physical stimuli, nesting and chewing materials, and highly palatable foods. Within each category, different items were included. A fixed number of objects per category were distributed into the cage on each configuration, which varied systematically twice per week during bed changes to avoid item repetitions within the same week (Table 1). Those objects that always remained in the cage were also rearranged (Table $\mathbf{1}$ ).

\section{Kinetics of Spontaneous Activity Within the EE Cage}

Once a week after bed changes, behavioral activity and USVs inside the EE cages were scored for $10 \mathrm{~min}$. One camera (GoPro Hero3, USA) was located at the frontal door (90 cm height) for monitoring the overall activity inside the cage. A second camera was located on the cage floor to monitor the activity at the lower levels. Five weekly sessions were recorded on the same days (between 7 and $9 \mathrm{am}$ ), starting with a baseline taken the first day of EE. The following behaviors were manually scored in segments of $10 \mathrm{~s}$ : running, climbing, jumping, rough-andtumble, chasing, material accumulation, moving objects, ripping objects or materials, foraging, eating, sniffing, and stretched-attempt posture. For USVs recordings, a microphone hung up from the center of the cage's ceiling at $40 \mathrm{~cm}$ above the first floor.

\section{OF Test}

Four rats, one from each group, were tested simultaneously on black Formica, square-wooden chambers $(55 \mathrm{~cm} \times 55 \mathrm{~cm} \times 40$ $\mathrm{cm}$ ) located in independent rooms illuminated with white dimmed light (10 \pm 1 lumens). Animals were individually placed in the middle of the arena and tested for $15 \mathrm{~min}$. Afterward, the apparatuses were cleansed with ethanol (70\%). Behaviors were video recorded (cameras at $80 \mathrm{~cm}$ height; GoPro Hero3-4, USA) for offline analysis. Locomotion was automatically scored using the video tracking system AnyMaze (version 5.1, Stoelting Co., Wood Dale, IL, USA) and reported as distance traveled in meters. The frequency of rearing and the duration of grooming were manually scored by trained observers (> 90\% inter-observer reliability) using Solomon Coder free software (version 17.03.22; https://solomoncoder.com/ download.php). Solomon Coder data was extracted using custom-made macros of Microsoft Office Excel. Rearing consisted of a bipedal posture $\left(>45^{\circ}\right.$ from the floor) where the animal extends its head upwards, executing a series of lateral movements using vibrissae to sense the surroundings. Positions $<45^{\circ}$ were excluded by being regarded as incomplete postures. We developed a grooming classification system based on its anatomical distribution and its levels of motor complexity (Rojas-Carvajal and Brenes, submitted). Based on its anatomical distribution, we classified grooming into three categories: cephalic (Figures 2.1-3: hand rubbing, face washing, unilateral and bilateral strokes), caudal (Figure 2.4: body liking and/or anus-genital licking), and sequential (Figures 2.1-4: chained events of cephalic and caudal grooming). Regarding grooming complexity, we observed that rats could use the hind paws to perform grooming sequences within each of the previous anatomical categories resulting in six possible grooming subtypes (Figure 2). When grooming was interrupted by locomotion (i.e., full displacement by using the four paws) or by any other behavior for $>8 \mathrm{~s}$, separated events were counted. If a rearing occurred within a grooming event, its time was discounted from the total grooming duration. Micro grooming $(<1 \mathrm{~s})$ events were also counted, but isolated scratching events were discarded (Figures 2.5a, b). Finally, USVs were recorded with a microphone placed $40 \mathrm{~cm}$ above the $\mathrm{OF}$ floor.

\section{Analysis of USVs}

USVs were monitored with UltraSoundGate Condenser Microphones (CM16; Avisoft Bioacoustics, Berlin, Germany) and recorded with Avisoft Recorder 2.7 software (sampling 
rate: $214,285 \mathrm{~Hz}$; format: $16 \mathrm{bit}$ ). High-resolution spectrograms (frequency resolution:.488 kHz, time resolution:.512 ms) were obtained after a fast Fourier transformation (512 FFT-length, $100 \%$ frame, Hamming window, 75\% time window overlap), by using the Avisoft SASLabPro 5.2 software. First, USVs were automatically detected using the Avisoft SASLabPro function of "whistle tracking" by adjusting the minimal duration, the hold time, and the peak amplitude. USVs emitted within a frequency range of $19-32 \mathrm{kHz}$ were considered as $22-\mathrm{kHz}$ USVs, and USVs between 33 and $96 \mathrm{kHz}$ were defined as $50-\mathrm{kHz}$ USVs (Brenes et al., 2016). Later, experienced observers manually filtered and selected the genuine USVs based on our own criteria reported elsewhere (Brenes and Schwarting, 2015; Brenes et al., 2016). Briefly, if two $50-\mathrm{kHz}$ elements were at least .048 s apart, two independent $50-\mathrm{kHz}$ calls were counted. A flat call was scored when peak-frequency changes within a single call element were equal to, or lower than $5 \mathrm{kHz}$. Any change in peak-frequency higher than $5 \mathrm{kHz}$ either within a single $50-\mathrm{kHz}$ element (e.g., the zigzag shape in trills calls) or between two or more overlapped 50-kHz USV elements (e.g., as in all step-calls) was considered as a modulation in peak frequency (FM). Accordingly, the following $50-\mathrm{kHz}$ calls subtypes were measured: 1) flats, 2) step-flats (all elements are flat with at least one overlapped element), 3) trills (one single element), and 4) step-trills (at least one element is a trill overlapped with one or more elements). As the rate of USVs is very variable between animals, the minute with the highest number of calls per animal was selected for USVs classification.

\section{Gene Expression Analysis}

Euthanasia was carried out by decapitation and brains were dissected on ice. The nucleus accumbens, the dorsal striatum, and the hippocampus were collected and prepared as previously reported (Sequeira-Cordero et al., 2013; Rojas-Carvajal et al., 2019). Oligonucleotides for BDNF, TrkB, and CREB were designed elsewhere (Rage et al., 2007; Liu et al., 2010), whereas primers for p250GAP and dnmt3a (p250GAP-F 5' ATGGATTTCAGGTGGGACTCTTC-3', p250GAP-R 5' GCTTTGTTGGGCGAGACTTCAT-3'; dnmt3a-F $5^{\prime}$ AGTCATCCGCCACCTCTTCG-3', dnmt3a-R 5' TCTCTCCGTCCTCTCGTTCTTG-3') were designed using the online tools Primer3 (http://bioinfo.ut.ee/primer3-0.4.0/), PrimerBLAST (https:/www.ncbi.nlm.nih.gov/tools/primer-blast/) and OligoAnalyzer (https://www.idtdna.com/pages/tools/ oligoanalyzer). Conditions for real-time reverse transcriptionquantitative polymerase chain reaction (RT-qPCR) were validated in a Rotor-Gene Q (QIAgen, Germany) according to published criteria (Raymaekers et al., 2009). Relative gene expression was determined by the comparative method with hypoxanthine phosphoribosyltransferase 1 (HPRT1) as a reference gene, which has been widely validated and used for the study of gene expression in rat brain tissues after several experimental conditions (Bonefeld et al., 2008; Santos and Duarte, 2008; Julian et al., 2014; Chapman and Waldenström, 2015; Sequeira-Cordero et al., 2019). In our hands, the use of other reference genes has been discouraged (e.g., actin or glyceraldehyde-3-phosphate dehydrogenase, GAPH) as they can be modified by the housing conditions (unpublished results). PCR reactions contained $4 \mu \mathrm{l}$ of 1:15 diluted cDNA, $5 \mu \mathrm{l}$ $2 \times$ Syber green (Fermentas, USA), and a final primer concentration of $75 \mathrm{nM}$ for HPRT1, CREB, and p250GAP, and 150, 300, and 200 $\mathrm{nM}$ for BDNF, TrkB, and DNMT3A, respectively; in a final volume of $10 \mu \mathrm{l}$. After an initial denaturation step at $95^{\circ} \mathrm{C}$ for $10 \mathrm{~min}$, amplification was performed with 40 cycles of denaturation at $95^{\circ} \mathrm{C}$ for $30 \mathrm{~s}, 45-\mathrm{s}$ annealing at $58^{\circ} \mathrm{C}$ for HPRT1 and $\operatorname{TrkB}, 63^{\circ} \mathrm{C}$ for p250GAP, and $64^{\circ} \mathrm{C}$ for BDNF, CREB, and DNMT3A, respectively; with an extension step at $72^{\circ} \mathrm{C}$ for $30 \mathrm{~s}$. Additionally, a melting curve analysis $\left(95^{\circ} \mathrm{C}\right.$ for $15 \mathrm{~s}, 60^{\circ} \mathrm{C}$ for $60 \mathrm{~s}$, and $95^{\circ} \mathrm{C}$ for $15 \mathrm{~s}$ ) was performed in order to confirm the specificity and primer dimmer absence. Samples were run in duplicates, and the mean values were used for further calculations. Each gene was run individually according to the sample maximization method (Hellemans et al., 2007), with each run including all housing groups. Non-template controls and minus RT controls were also included in order to exclude the possibility of genomic DNA contamination. The threshold cycle $(\mathrm{Ct})$ was calculated using the Rotor-Gene Q Series Software (QIAgen, Germany). mRNA levels were reported both as $2^{-\Delta \mathrm{Ct}}$ and as normalized values centered around the SH5 group mean (i.e., 100\%) expressed in percentages (see Figure 8).

\section{Statistical Analysis}

All the analyses were done with IBM SPSS v21 software (IBM, USA). Data were expressed as mean \pm standard error of the mean (SEM). The parameters measured in the EE cage were the following: locomotor activity (running, galloping, jumping, and climbing); cage exploration (stretch-attempt posture and sniffing); material/items remodeling (digging, gnawing/ chewing, material accumulation, material ripping, and item moving); eating; foraging; social interaction (rough-and-tumble play and chasing); and USVs. When two or more behaviors belonged to one category, they were summed up to be compared with each other. These variables were analyzed using a four-way analysis of variance (ANOVA) with behavior (the different categories), housing (i.e., CEE and REE), minutes (i.e., 1-10), and weeks (i.e., 1-5) as factors. In the OF, locomotion (distance traveled), rearing frequency, grooming duration (total and its subtypes), and USVs (total number and its subtypes) were analyzed with mixed multivariate ANOVAs with groups (i.e., SH2, SH5, CEE, and REE) and days (i.e., 1-4) as between-subject factors and minutes (i.e., 1-15) as a within-subjects factor. When both $\mathrm{SH}$ and $\mathrm{EE}$ groups were quite similar between each other, a main effect of housing (i.e., SH vs. EE) was estimated with oneway ANOVA. The percentage of USVs subtypes was analyzed within each group with one-way ANOVA with call subtype (i.e., flat, step-flats, trills, and step-trills) as a within-subjects factor. Protected, Fisher's Least Significant Difference (LSD) post hoc test was used for multiple comparisons among groups. Bonferroni's adjustment was applied for multiple comparisons among minutes and days, when appropriate. Results of the gene expression were analyzed with one-way ANOVA with groups (i.e., SH5, CEE, and REE) as the between-subject factor followed 
by controlled, planned contrasts. For all analyses, the effect size was estimated with the partial eta-squared $\left(\eta_{p}^{2}\right)$ coefficient and the statistical significance was defined as $P<.05$.

\section{RESULTS}

\section{Experiment 1}

\section{REE Increases the Activity and the Rate of} Appetitive USVs in the Cage

Several lines of evidence have shown that restricted or unpredictable access to rewarding stimuli (e.g., food or drugs of abuse) increases both incentive motivation attributed to reward-predicting cues and approaching and consummatory responses toward the reward (Anselme et al., 2013; Brenes and Schwarting, 2014; Schultz, 2016; Kreisler et al., 2018). Considering that laboratory rodents prefer larger and complex cages, social contact, and exercise (Olsson and Dahlborn, 2002; Van Loo et al., 2003; Heyse et al., 2015), we assume that EE is rewarding for rodents. Rats emit high-frequency (e.g., $50-\mathrm{kHz}$ ) USVs in social (e.g., mating and rough-and-tumble play) and non-social rewarding situations (e.g., brain stimulation of reward centers and administration of psychostimulants) (Burgdorf et al., 2000; Williams and Undieh, 2010; Pereira et al., 2014; Brenes and Schwarting, 2015). Out of the different $50-\mathrm{kHz}$ calls, the FM subtypes (e.g., step-flats, step-trills, and trills) are indicative of a high, positive affective state (Burgdorf et al., 2007; Burgdorf et al.,
2008; Burgdorf et al., 2011). Thus, we hypothesized that unpredictable, restricted access to EE would intensify the incentive value of the $\mathrm{EE}$ cage, increasing exploratory activity and $50-\mathrm{kHz}$ calls during the first minutes of EE exposure. To test this hypothesis, we monitored the behavioral and USVs responses displayed by CEE and REE animals within the EE cage throughout the housing weeks. We found that physical activity and cage exploration (i.e., sniffing, rearing, and stretchattend posture) were the most frequent behaviors during the whole EE period (behaviors: $F_{(2.15,1285.05)}=1653.53, p=.001$, $\eta_{p}^{2}=.74$; Figure 3G). Both EE groups progressively increased locomotor activity across minutes and weeks, but REE animals did it to a greater extent (groups ${ }^{\star}$ minutes ${ }^{\star}$ weeks: $F_{(36,500)}=$ 3.44, $p=.001, \eta_{p}^{2}=.20$ ) (Figure 3A). Cage exploration decreased over minutes only in REE rats (groups * minutes: $F_{(9,500)}=3.72$, $\left.p=.001, \eta_{p}^{2}=.06\right)$, but progressively increased over weeks in both EE groups, especially in CEE animals (groups ${ }^{\star}$ weeks: $F_{(4,500)}=$ 15.60, $p=.001, \eta_{p}^{2}=.11$ ) (Figure 3B). Remodeling and modifying the materials and items of the EE cage was one of the behaviors in which the group differences were the greatest, with such activity being increasingly higher in REE rats (groups * minutes * weeks: $\left.F_{(36,500)}=3.44, p=.001, \eta_{\mathrm{p}}^{2}=.20\right)$ (Figure 3C). The frequency of foraging (groups $*$ minutes $*$ weeks: $F_{(36,500)}=2.27, p=.001, \eta_{\mathrm{p}}^{2}=.14$ ) (Figure 3D) and eating (group ${ }^{\star}$ minutes ${ }^{\star}$ weeks: $F_{(36,500)}=3.94, p=.001, \eta_{p}^{2}=.22$ ) (Figure 3E) was very low during the evaluation period. These behaviors increased slightly in both groups and, in the case of
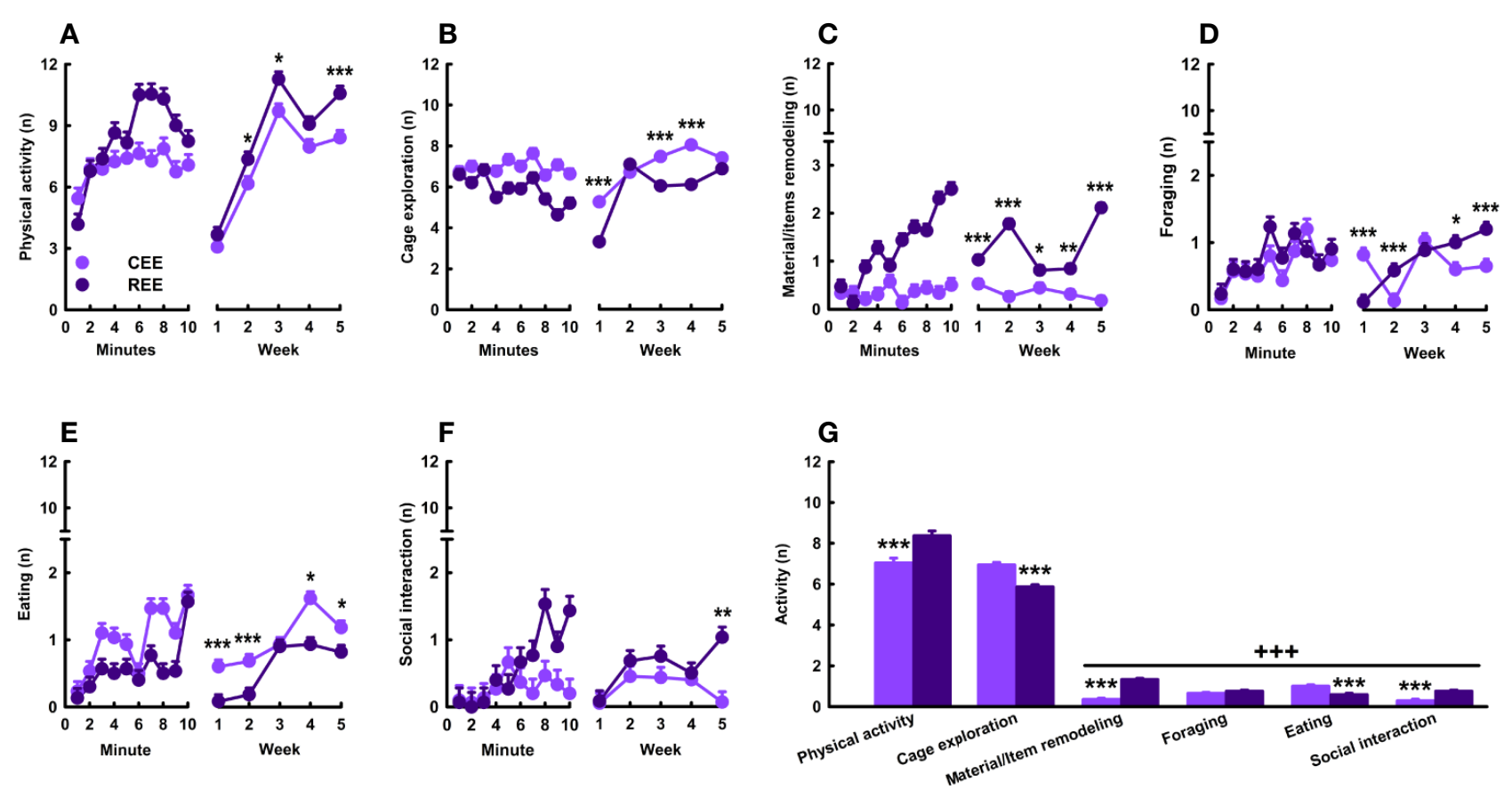

FIGURE 3 | Behavioral kinetics in the environmental enrichment cage. Physical activity (A), cage exploration (B), material/items remodeling (C), foraging (D), eating (E), and social interaction (F) displayed over minutes (left panels) and weeks (right panels). Cumulative average activity (G). Data correspond to frequencies expressed as mean \pm SEM. CEE, continuous environmental enrichment; REE,restricted and unpredictable environmental enrichment. Single, pairwise-comparisons between groups: ${ }^{\star} p<.05 ;{ }^{\star \star} p<.01 ;{ }^{\star \star \star} p<.001$. Comparison between behaviors: ${ }^{+++} p<.001$. 
eating, it was significantly higher in CEE rats. Social interaction increased per minute and over weeks, but only in REE rats (Figure 3F). The CEE animals, in contrast, showed irregular frequencies per minute with an inverted $U$-shaped pattern when analyzed through the weeks (groups ${ }^{\star}$ minutes ${ }^{\star}$ weeks: $F_{(36,500)}=$ $\left.1.70, p=.01, \eta_{p}^{2}=.11\right)$. Once the animals entered the EE cage, the rate of USVs started to increase progressively in both conditions (minutes: $F_{(9,99)}=2.143, p=.05, \eta_{p}^{2}=.19$ ) (Figure 4A, right panel). When comparing by groups, the total number of $50-\mathrm{kHz}$ calls was higher in REE rats (groups: $F_{(1,99)}=89.075, p=.0001$, $\eta_{\mathrm{p}}^{2}=.50$ ) (Figure 4A, left panel). Over weeks, the rate of USVs showed an inverted U-shaped pattern, which peaked at week two and then decreased linearly in the following weeks (weeks: $F$ $\left.(4,99)=15.790, p=.0001, \eta_{p}^{2}=.41\right)$, with the REE rats showing a consistently higher call rate throughout the housing period (groups * weeks: $F_{(4,99)}=6.070, p=.0001, \eta_{p}^{2}=.21$ ) (Figure 4A, middle panel). When analyzing the USVs subtypes, REE rats emitted more FM calls, especially the subtypes with higher peak modulations (i.e., trills and step-trills) (Figure 4B).

To our knowledge, this is the first study describing the kinetics in overt behavior and USV s within the EE cage. This study also provides the first empirical evidence that the motivation to "get environmentally enriched" can be increased by restricting access to the cage unpredictably. Based on the behavioral data, it seemed that REE rats tried to make the most out of their limited time in the cage, which in turn changed progressively the type and the combination of activities they chose to do. During these $10 \mathrm{~min}$, REE rats spent their time traveling around and interacting actively with the cage, the materials, and other rats (Figures 3A, C, F). A similar study also found that, as compared to $\mathrm{SH}$ rats, $\mathrm{EE}$ counterparts displayed higher levels of social and non-social activity within the cage, with the level of such activities being even higher in the group enriched with natural items than in the group enriched with artificial ones (Lambert et al., 2016). As our protocol was designed to enhance the interaction with natural, ethologically relevant stimuli and social partners in a sufficiently large space, the distribution and selection of activities made by the REE rats may shed light on which ethological needs are more urgent to be fulfilled when time is limited. The low levels of eating behavior in REE rats could be the result of the competing motivation to perform different behaviors in addition to the net effect of exercise on appetite, which is known to reduce food intake throughout the regulation of several neural messengers, such as insulin, ghrelin, and the corticotropin-release factor (Kawaguchi et al., 2005; Ebal et al., 2007). Based on the level of activity and USV displayed in the cage, it can be concluded that such an environment was highly rewarding for all animals, but to a greater extent for those with restrict and unpredictable access to the cage. For instance, the between-group differences in the number of $50-\mathrm{kHz}$ calls were 2.28 and 3.67 -fold higher in the REE group, with the lowest call rate of the REE rats being even higher than the highest point of the CEE rats. Considering that $50-\mathrm{kHz}$ calls may signal a state of appetitive incentive motivation (Brenes and Schwarting, 2014; Brenes and Schwarting, 2015), the differences in USVs suggest that unpredictable and restricted access to the EE cage could have extended and enhanced the rewarding properties of the EE stimuli. In support to the latter, we have previously found that rats trained to run a runway to enter a locked running wheel, displayed almost the same latencies and rates of $50-\mathrm{kHz}$ calls than those with the unlocked wheel, suggesting that the structural features of the wheels were equally attractive for both groups (Heyse et al., 2015). Once the rats with the unlocked wheel exercised, the rate of $50-\mathrm{kHz}$ calls increased substantially, indicating that the activity on its own was also rewarding for them (Heyse et al., 2015). As the REE rats were housed in groups of five during the non-EE period, entering the EE cage offered the opportunity for interacting with non-cagemates rats, which could have also been a great source of reward. The higher call rate and the larger

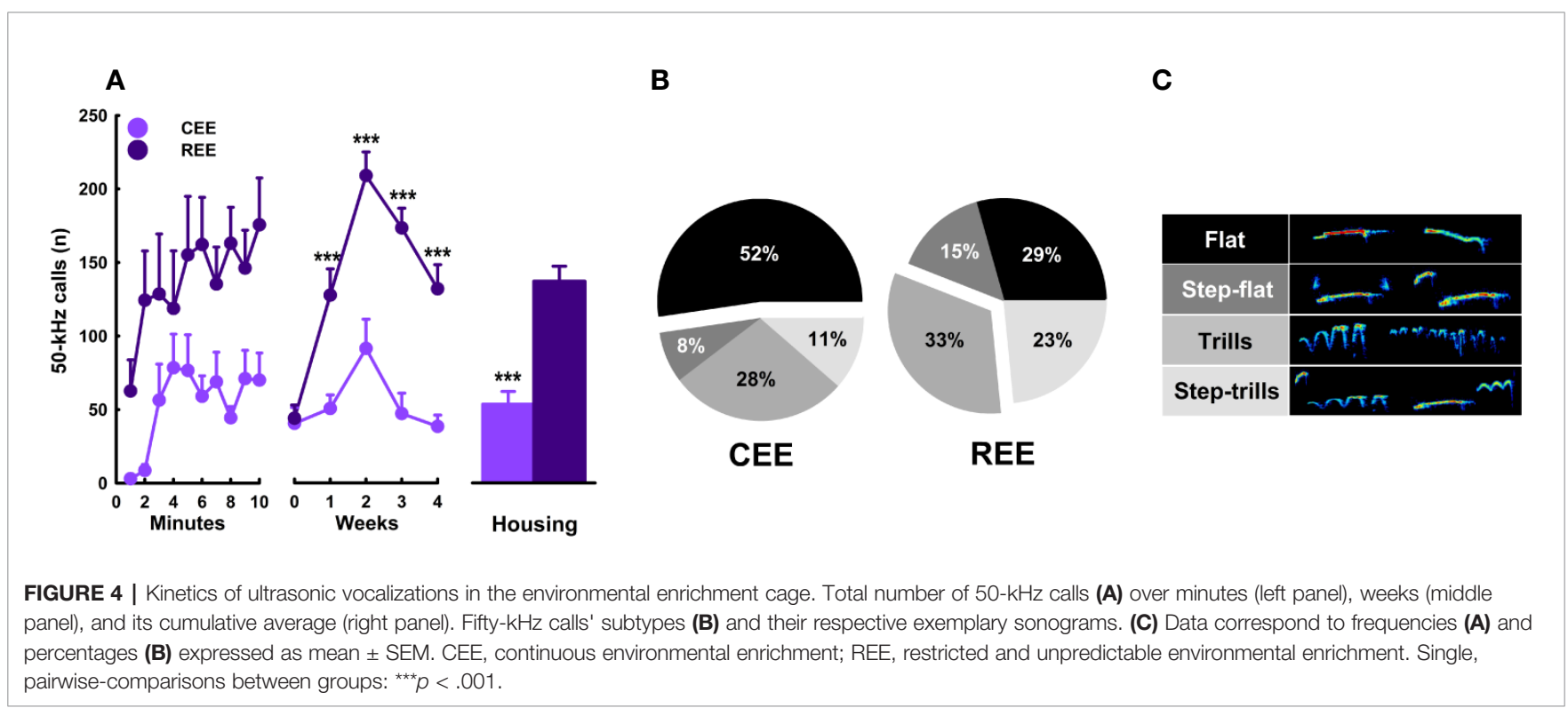

Frontiers in Pharmacology | www.frontiersin.org 
percentage of FM USVs seen in REE rats may have resulted from social interactions perceived as more appetitive, although the level of social contact was similar between groups during the first weeks. In fact, rough-and-tumble play and social contact are known to elicit high rates of $50-\mathrm{kHz}$ calls, especially in juveniles (Knutson et al., 1998; Burgdorf et al., 2008). As the call rate increased until week two and then decreased in the following weeks (Figure 4A, middle panel), we might suppose that the hedonic and motivational properties associated with novelty, exercise, and social interaction required 2 weeks to reach the highest level. Afterward, the affective response to the EE cage seemed to decrease in all animals, despite the efforts made to maintain the novelty and complexity of such an environment. Complementarily, the reduction in call rate seen on weeks three and four may be associated with an age-dependent effect on social interaction-induced $50-\mathrm{kHz}$ calls. Time spent interacting with conspecifics is known to reduce with age, as well as the emission of and the behavioral response to $50-\mathrm{kHz}$ calls (Salchner et al., 2004; for review, see Seffer et al., 2014). Therefore, the call rate may have reduced, as animals were moving away from the age where rough-and-tumble play is highly frequent (Burgdorf et al., 2008; Seffer et al., 2014). Based on our results, it is unlikely that the changes on USV s constitute a mere byproduct of other behavioral activities. The analysis of USVs provided meaningful insights about the subjective motivational and emotional states of the rat in response to the EE, which might not be otherwise obtained.

\section{REE Equals the CEE-Induced Enhancements in Novelty Habituation}

We have found that restricted the unpredictable access to $\mathrm{EE}$ increased the interaction with social and non-social stimuli and the affective responses to them. Thus, we wondered whether such a more intense and motivated EE experience could translate into a noticeable behavioral outcome. Because the decline of locomotion and rearing in the $\mathrm{OF}$ is one of the most consistent, robust, and well-replicated findings of $\mathrm{EE}$ in rats (Elliott and Grunberg, 2005; Brenes Sáenz et al., 2006; Brenes et al., 2008; Brenes et al., 2009; Turner and Burne, 2014; Urakawa et al., 2014; Brenes et al., 2016; Rojas-Carvajal et al., 2018; Sampedro-Piquero et al., 2018), we used this paradigm as a behavioral readout of the effects of our housing conditions. As expected, all animals habituated to the OF as noted by the reduction of locomotion (minutes: $F_{(10.87,1827.37)}=253.17$, $p=.0001, \eta_{\mathrm{p}}^{2}=.60 ;$ minutes $*$ days: $F_{(32.63,1827.37)}=2.47$, $p=.0001, \eta_{\mathrm{p}}^{2}=.04$ ) (Figure 5A) and rearing frequency (minutes: $F_{(11.67,1960.63)}=61.72, p=.0001, \eta_{p}^{2}=.27$; minutes * days: $\left.F_{(35.01,1960.63)}=2.08, p=.0001, \eta_{p}^{2}=.04\right)$ (Figure 5B) throughout the minutes and days. A detailed analysis revealed that such a reduction on OF activity occurred only in the EE groups, especially after the OF2 for locomotion (minutes * groups: $F_{(32.63,1827.37)}=2.37, p=.0001, \eta_{\mathrm{p}}^{2}=.04$; minutes * days * groups: $\left.F_{(97.90,1827.37)}=1.43, p=.002, \eta^{2}{ }_{p}=.07\right)$, and the OF3 for rearing (minutes ${ }^{\star}$ groups: $F_{(35.01,1960.63)}=2.08, p=.0001$, $\eta_{\mathrm{p}}^{2}=.04 ;$ minutes $*$ days $*$ groups: $F_{(105.03,1960.63)}=1.37$, $\left.p=.009, \eta_{p}^{2}=.07\right)$. When comparing locomotor activity between the housing conditions, we found that animals in both EE conditions traveled less distance than their SH counterparts on days 2 to 4 (housing: all $p$-values $<.001, \eta^{2}{ }_{\mathrm{p}}=.35-.49$; LSD: $p<$ .05) (Figure 5A, middle panel). In addition, SH5 rats traveled more distance than SH2 animals on days 2 and 4 (all $p$-values < .05 ; LSD: $p<.05$ ), with the SH5 group also differing from each EE group on all testing days (all $p$-values $<.05$ ). Consequently, the cumulative distance traveled was lower in both EE groups than in both $\mathrm{SH}$ groups and also the $\mathrm{SH} 2$ and $\mathrm{SH} 5$ groups differed from each other (groups: $F_{(3,180)}=29.621, p=.0001, \eta_{p}^{2}=.33$ ) (Figure

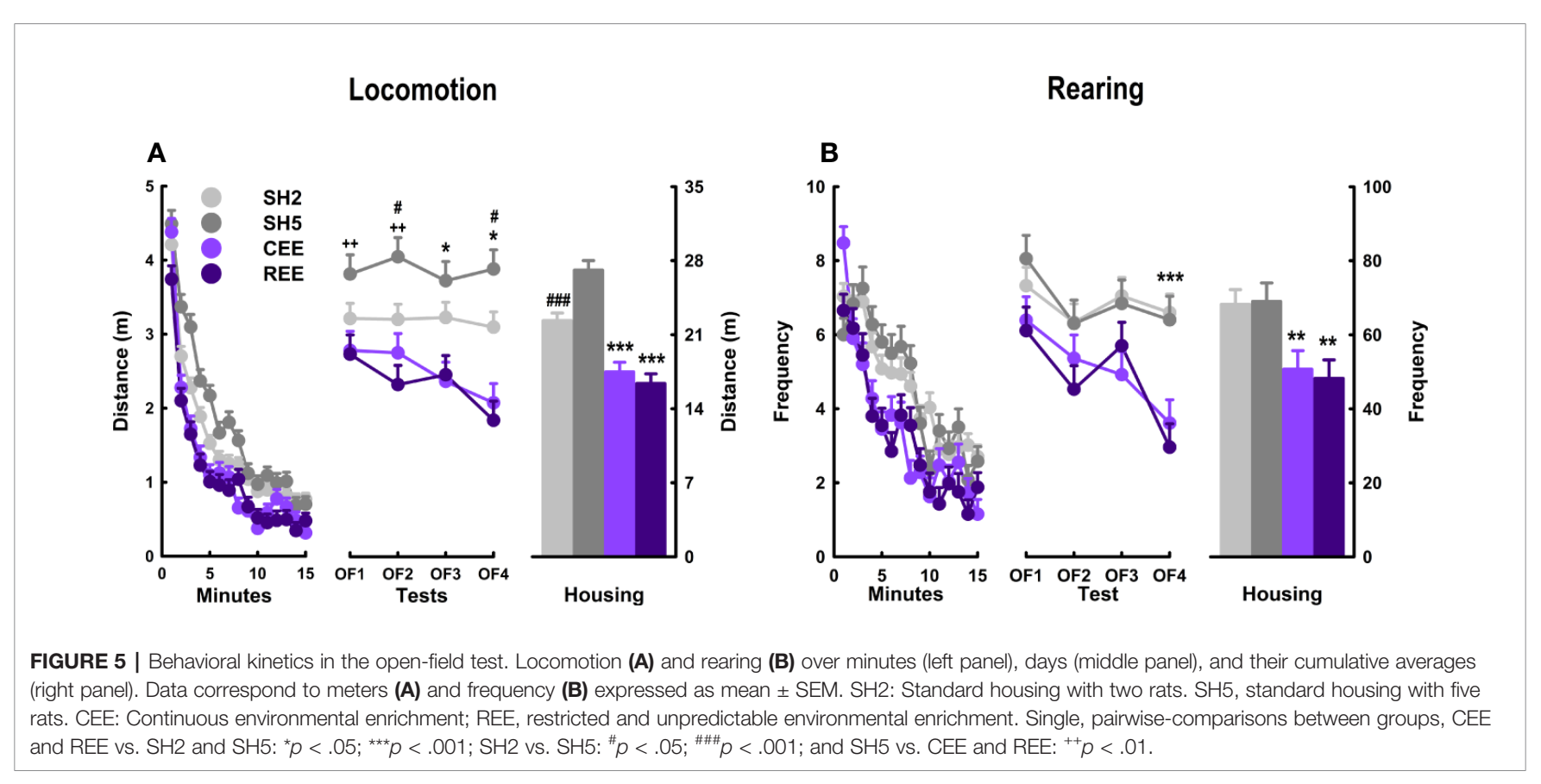


5A, left panel). When analyzing rearing, both EE groups showed the lowest levels throughout the testing period (housing: all $p$ values <.001, $\eta_{p}^{2}=.13-.49$; LSD: $p<.05$ ), but single-group differences were only observed on OF4, where each $\mathrm{SH}$ group differed from each EE group (LSD: $p<.05$ ) (Figure 5B, middle panel). The cumulative rearing frequency was lower in both $\mathrm{EE}$ groups than in both $\mathrm{SH}$ groups with no further differences between the $\mathrm{SH}$ and $\mathrm{EE}$ groups (groups: $F_{(3,180)}=12.319$, $p=.0001, \eta_{p}^{2}=.17$ ) (Figure 5B, right panel).

The OF behavior of both EE groups was almost identical, suggesting that being enriched only $50 \%$ of the total period did not compromise the effects of EE in this test. Evidence from short and restricted EE protocols have revealed neurobehavioral improvements with $3 \mathrm{~h}$ /day over 3 weeks (Stein et al., 2016), 2 h/day for 30 days (Widman and Rosellini, 1990), and $2 \mathrm{~h} /$ day for 40 days (Widman et al., 1992), suggesting that partial EE is sufficient to induce the expected reduction of locomotion and rearing in the OF (i.e., enhanced novelty habituation). Alternatively, the restricted and unpredictable access to the cage may have potentiated the stimulating effects of $\mathrm{EE}$ compensating the $50 \%$ of the time they were not in the $\mathrm{EE}$ cage. The EE-induced improvements of non-associative learning agree with previous evidence obtained in the OF (Elliott and Grunberg, 2005; Brenes Sáenz et al., 2006; Brenes et al., 2008; Brenes et al., 2009; Brenes et al., 2016; Rojas-Carvajal et al., 2018; Sampedro-Piquero et al., 2018; Mora-Gallegos and Fornaguera, 2019) and with findings of improved performance in episodic and spatial memory tasks (Sampedro-Piquero et al., 2013; Turner and Burne, 2014; Mora-Gallegos et al., 2015; Brenes et al., 2016; Mármol et al., 2017). From a cognitive perspective, it is interpreted that the faster the decline of the activity, the faster the processing of novel and threatening stimuli (Kempermann et al., 2002; Hughes and Collins, 2010). From an emotional perspective, it is assumed that $\mathrm{EE}$ animals become less responsive to mild stressors, as EE typically produces a faster reduction in exploratory and defensive responses in anxiety and fear paradigms (Hendriksen et al., 2010; Mitra and Sapolsky, 2012; Ashokan et al., 2016; Koe et al., 2016). Integrating both perspectives, EE is thought to facilitate the extraction of information about the likely sources of threats during the first testing minutes, which is sufficient to reduce arousal and defensiveness within the test. With repeated exposures, this learning generalizes to the same or similar contexts (Rojas-Carvajal et al., 2018; Rojas-Carvajal and Brenes, submitted). As expected, between-days habituation was currently observed in our EE rats (Figure 5). In SH rats, however, no such an effect was detected for locomotion, and in the case of rearing, only a small reduction was observed from OF1 to OF2. We attributed this lack of effects on the handling procedure employed in this experiment. The $\mathrm{SH}$ rats were handled more frequently than usual to equate the manipulation received by the EE groups, as they were put in and out of the EE cage several times per week. Therefore, handling may have reduced the behavioral reactivity to the OF until a point where no further reductions were possible. In agreement with our results, lengthy handling procedures (1-6 weeks) have been found to reduce locomotor responsiveness to the OF (Hirsjärvi and Väliaho, 1995; Schmitt and Hiemke, 1998;
Costa et al., 2012). In unhandled rats, we have observed a more pronounced activity decay in non-EE rats tested exactly as in this experiment (i.e., on four consecutive 15-min tests) (Rojas-Carvajal et al., 2018), with the levels of locomotion and rearing being $20 \%$ and $\sim 10 \%$ higher than those reported here, respectively. Finally, it is worth mentioning that the $\mathrm{SH}$ groups differed to each other on locomotor activity, with the $\mathrm{SH} 5$ group traveling more distance than the $\mathrm{SH} 2$ group (Figure 5A). We have initially expected that the social enrichment provided by the SH5 housing would reduce or at least equate the behavioral reactivity of $\mathrm{SH} 2$ rats. However, we found quite the opposite. We attribute this effect to the mild stress provoked by cage overcrowding. Over time, the cage area became proportionally smaller relative to the animal size, something that may have occurred to a lesser extent in the $\mathrm{SH} 2$ group. We have found the same results in the OF when comparing rats housed in groups of six or pairs (Brenes et al., 2016).

\section{EE Differentially Affects Grooming Subtypes and Kinetics in the OF}

Besides locomotion and rearing, grooming is one of the most responsive $\mathrm{OF}$ parameters to $\mathrm{EE}$. In consequence, it constitutes a suitable candidate for the analysis of particular differences induced by our EE protocols. Regarding its association with stress, two seemingly opposite interpretations have been proposed: 1) grooming is a direct marker of distress emitted as a byproduct of other defensive behaviors; or 2) grooming is a stress-coping response that facilitates emotional de-arousal (Spruijt et al., 1992; van Erp et al., 1994; Brenes et al., 2009; Kalueff et al., 2016; Estanislau et al., 2019). However, the analysis of grooming structure and time course has revealed that both interpretations correspond to different phases of the same behavioral process (Brenes et al., 2009; Kalueff et al., 2016; Rojas-Carvajal et al., 2018; Estanislau et al., 2019; RojasCarvajal and Brenes, submitted). To integrate these perspectives, we have proposed that bursts of short cephalic grooming are more likely associated with arousal and distress since they appear at the beginning of tests and then decrease progressively along with exploration and risk-assessment. In contrast, longer and more complex grooming sequences should be part of a de-arousal inhibition system restoring emotional homeostasis, as they increase when habituation is taking place and defensive behaviors are less prominent (Brenes Sáenz et al., 2006; Brenes et al., 2009; Rojas-Carvajal et al., 2018; RojasCarvajal and Brenes, submitted). Based on this evidence, we analyzed the structure and kinetics of grooming. Regarding the total grooming time, we found that both EE groups showed the highest scores (groups: $F_{(3,992)}=42.03, p=.0001, \eta_{p}^{2}=.11$ ) (Figure 6A). A detailed analysis of its structure revealed differences between the subtypes (subtypes: $F_{(5,992)}=279.77$, $p=.0001, \eta_{\mathrm{p}}^{2}=.59$ ), which also varied between groups (subtypes * groups: $\left.F_{(15,992)}=44.32, p=.0001, \eta_{\mathrm{p}}^{2}=.40\right)$. Such an interaction between subtypes and groups remained throughout the minutes (subtypes ${ }^{\star}$ groups ${ }^{\star}$ minutes: $F_{(210,13888)}=2.44, p=.0001, \eta^{2} \mathrm{p}$ $=.04$ ) and days (subtypes ${ }^{\star}$ groups ${ }^{\star}$ minutes ${ }^{\star}$ days: $F_{(630,13888)}=$ $\left.1.27, p=.0001, \eta_{p}^{2}=.06\right)$. Among the different subtypes, cephalic grooming and sequential grooming with variations were the 
Total grooming

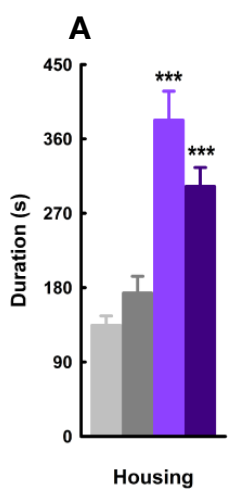

Cephalic grooming

\section{B}

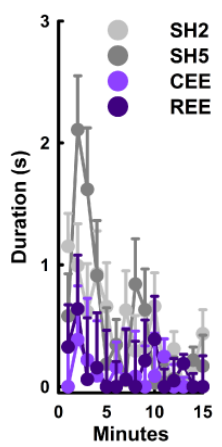

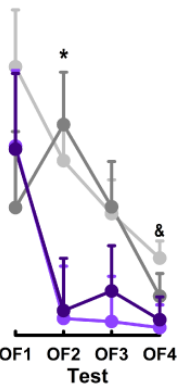

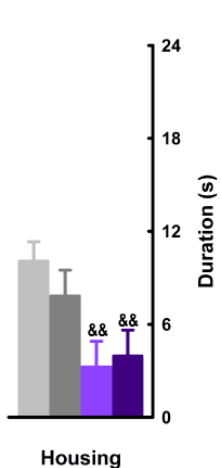

Sequential grooming with variations

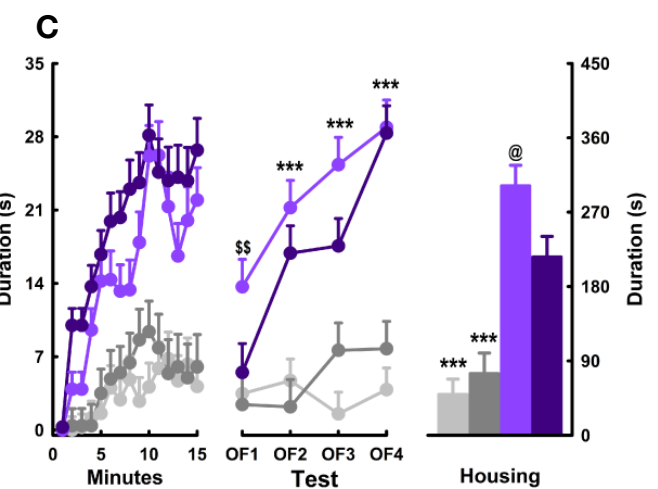

FIGURE 6 | Grooming kinetics and subtypes in the open-field test. Total grooming (A), cephalic grooming (B), and sequential grooming with variations (C) over minutes (left panel), days (middle panel), and their cumulative averages (right panel). Data correspond to seconds expressed as mean \pm SEM. SH2: Standard housing with two rats. SH5, standard housing with five rats. CEE, continuous environmental enrichment; REE, restricted and unpredictable environmental enrichment. Symbols correspond to single, pairwise-comparisons between groups. CEE and REE vs. SH2 and SH5: ${ }^{*}<<.05 ;{ }^{\star \star \star} p<.001$. SH2 vs. CEE and REE: ${ }^{\&} p<.05 ;{ }^{\& \&} p<.01$. CEE vs. REE: ${ }^{@} p<.05$. CEE vs. REE, SH2 and SH5: ${ }^{\$ \$} p<.01$.

most responsive subtypes to the effects of housing (groups: $\left.F_{(3,180)}=58.17, p=.0001, \eta_{\mathrm{p}}^{2}=.51\right)$, following quite the opposite kinetics within the $\mathrm{SH}$ and the $\mathrm{EE}$ groups (Figures 6B, C). On one hand, all animals showed high but irregular levels of cephalic grooming (e.g., in OF1), which decreased throughout the minutes (minutes: $F_{(15,180)}=2.46, p=.003, \eta_{\mathrm{p}}^{2}=.18$ ) and days (days: $F_{(3,120)}=6.61, p=.001, \eta_{p}^{2}=.14$ ) (Figure 6B, left and middle panel). In $\mathrm{SH}$ rats, cephalic grooming reduced linearly from OF1 to OF4, whereas in EE counterparts, it abruptly declined from OF1 to OF2 and then remained unchanged thereafter (Figure 6B, middle panel). When compared by groups, it was found that EE groups showed less cephalic grooming than both $\mathrm{SH}$ groups in $\mathrm{OF} 2$, and the $\mathrm{SH} 2$ group in OF4 (all $p$-values $p<.05, \eta_{\mathrm{p}}^{2}=.18-.24$; LSD: $p<.05$ ). In consequence, $\mathrm{SH}$ groups had the highest levels of cephalic grooming throughout the days (groups: $F_{(3,180)}=7.19$, $p=.0001, \eta_{p}^{2}=.12$; LSD: $p<.05$ ) (Figure 6A, right panel). On the other hand, sequential grooming with variations increased gradually over minutes (minutes: $F_{(15,180)}=15.71, p=.0001$, $\left.\eta_{\mathrm{p}}^{2}=.59\right)$ and days (days: $\left.F_{(3,120)}=11.96, p=.0001, \eta_{\mathrm{p}}^{2}=.18\right)$. A detail inspection revealed that such an effect occurred exclusively in both EE groups, which showed a linear increase throughout the days (minutes ${ }^{\star}$ groups: $F_{(21.58,1193.93)}=2.27, p=.001, \eta_{\mathrm{p}}^{2}$ $=.04$; days ${ }^{\star}$ groups: $F_{(9,120)}=3.24, p=.001, \eta_{p}^{2}=.15$ ) (Figure 6C, left and middle panel). In OF1, the CEE group showed higher levels of sequential grooming with variations than the other groups (LSD: $p<.01$ ), being the first OF parameter in which the EE groups differed from each other (Figure 6C, middle panel). On the following days, however, such differences were no longer observed as grooming increased gradually in REE rats until reaching the levels of that in CEE counterparts. Consequently, both CEE and REE groups differed from both $\mathrm{SH}$ groups from OF2 to OF4 (all $p$-values $p<.0001, \eta_{p}^{2}=.50-.65$; LSD: $p<.05$ ).
The SH5 rats had descriptively higher levels of sequential grooming with variations than $\mathrm{SH} 2$ rats, which were marginal on OF3 and OF4. When comparing among groups the cumulative time spent on this subtype, the same pattern was observed: each EE group differing from each $\mathrm{SH}$ group (groups: $F_{(3,178)}=44.97, p=.0001, \eta_{\mathrm{p}}^{2}=.43$; LSD: $p<.05$ ) (Figure 6C, right panel).

So far, the behavioral changes induced by both EE protocols on traditional OF parameters were alike. The analysis of grooming structure and time course, however, did reveal particular grooming differences between the EE groups. Supporting our interpretations of the grooming subtypes, we found that cephalic grooming was higher at the beginning of the test, declined with repeated testing, and was lower in EE rats. In contrast, sequential grooming with variations gradually increased with repeated exposures being 2- and 3-fold higher in REE and CEE rats than in SH counterparts, respectively. Contrary to locomotion or rearing, the REE rats required repeated $\mathrm{OF}$ exposures to show the same grooming levels observed in the CEE rats, suggesting that in this particular domain, the REE protocol did not fully compensate for the reduced time into the EE cage. EE-induced higher levels of grooming have also been observed in other conditioned and unconditioned anxiety tests (Turner and Burne, 2014; Sparling et al., 2018; Mora-Gallegos and Fornaguera, 2019). Because EE resembles the natural habitat of the rat and reduces the stress of captivity (Swaisgood, 2007; Tarou and Bashaw, 2007), it is improbable that the high levels of grooming observed in EE animals constitute a distress or displacement response indicative of negative emotionality. Instead, the exposure of rats to EE seems to promote a behavioral phenotype in which both defensive and non-defensive responses to stress are not only equally present but also highly efficient (Mitra and Sapolsky, 
2012; Estanislau et al., 2019). In this line, specific grooming subtypes should confer particular ethological benefits by helping animals to restore emotional homeostasis and to gradually disengage from defensive responses when they are no longer required (Rojas-Carvajal et al., 2018). We are aware that distress and captivity may induce pathological forms of grooming by excess (i.e., nail-biting, barbering, and stereotypies) or defect (e.g., porphyrin accumulation or dirt accumulation in the fur). However, neither of these signs was observed in our EE rats. As grooming abnormalities have increasingly become a key marker in preclinical models of Autism-spectrum disorder (for review, see Bernard et al., 2015; Kalueff et al., 2016; Zhang et al., 2017), Obsessive-compulsive disorder (Hajheidari et al., 2017), Parkinson disease (Paumier et al., 2013), and Huntington's disease (Tartaglione et al., 2016), EE could be used in these models as a treatment to prevent or restore aberrant forms of grooming (Swaisgood, 2007).

\section{EE Reduces Total Calling While Shifting the USVs Subtypes in the OF}

Spontaneous $50-\mathrm{kHz}$ calls, especially the flat subtypes, are usually emitted when animals are transiently separated from conspecifics and placed alone in a testing context (Wöhr et al., 2008; Natusch and Schwarting, 2010; Brenes and Schwarting, 2014; Brenes et al., 2016). Flat calls appear at high rates at the beginning of the test and decay over time (Wöhr et al., 2008). This type of USVs is thought to serve both an exploratory and communicative function to maintain or (re)establish social contact and to reduce intra-specific aggression (Wöhr et al., 2008; Natusch and Schwarting, 2010; Brenes et al., 2016). FM 50-kHz calls, such as step-flats, trills, and step-trills, occur at a lower rate during exploratory activities, but appear prominently during social (e.g., rough-and-tumble play, mating, social contact) and non-social rewarding situations (e.g., psychostimulants, food, exercise) (Knutson et al., 1998; Burgdorf et al., 2008; for

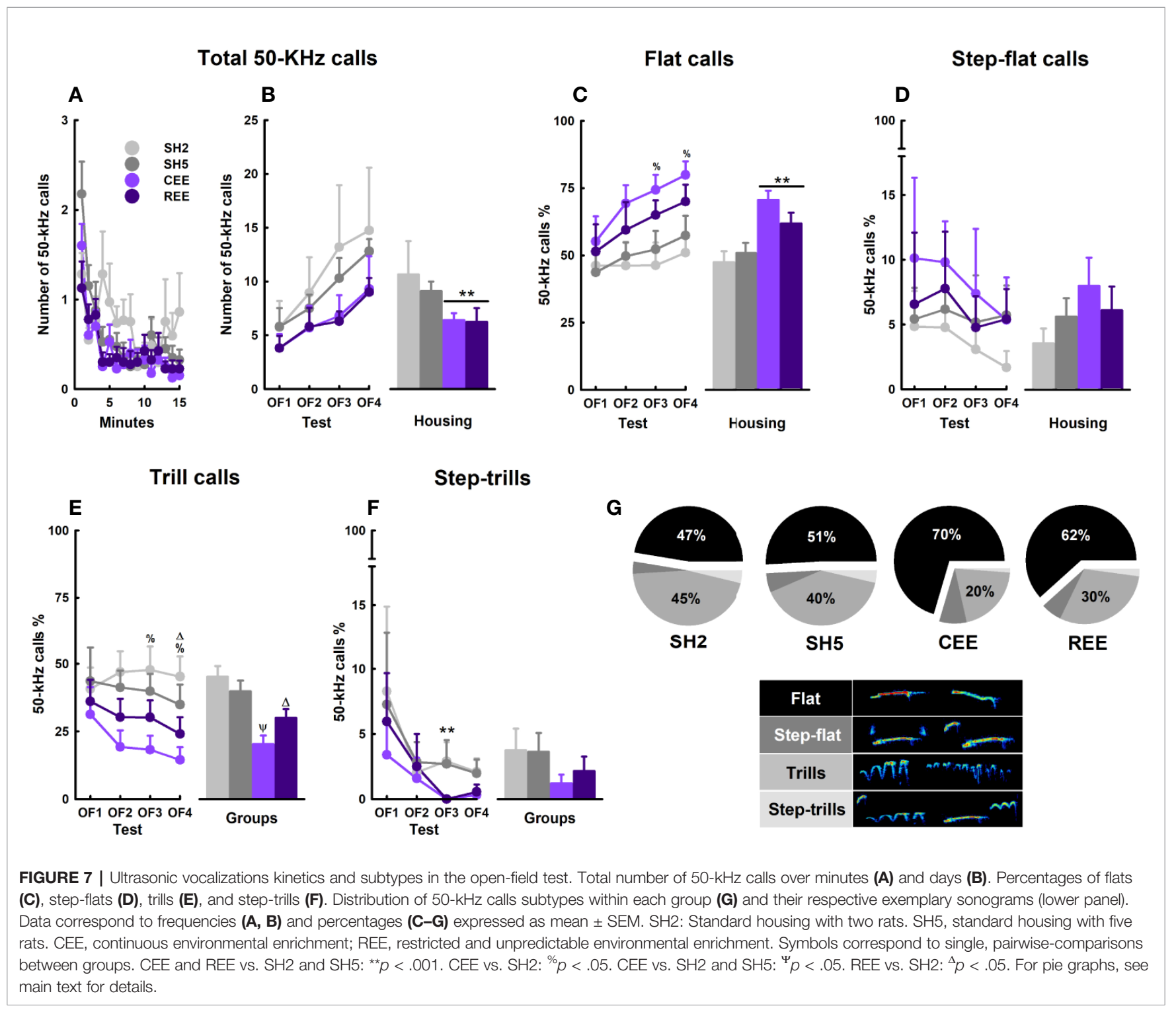


review, see Brudzynski, 2013). Besides its effects on exploration, risk-assessment, and emotional self-regulation, EE is known to modulate the emission of USVs in the OF (Brenes et al., 2016). To test whether our EE protocols affect prosocial communication, we analyzed the total number of USVs and the call profile during the OF sessions. We found that the total number of $50-\mathrm{kHz}$ calls decreased over minutes (minutes: $\left.F_{(6.07,1068.319)}=7.12, p=.0001, \eta_{p}^{2}=.04\right)$ (Figure 7A) but increased throughout the testing days (days: $F_{(3,176)}=2.89$, $p=.004, \eta_{\mathrm{p}}^{2}=.05$ ), especially when comparing OF1 and OF4 (Bonferroni: $p<.05$ ) (Figure 7B, left panel). Although both $\mathrm{EE}$ groups showed descriptively lower $50-\mathrm{kHz}$ calls on each $\mathrm{OF}$, the within-groups variability impeded reaching the significance level. On the cumulative number of $50-\mathrm{kHz}$ calls, both EE groups showed lower levels than SH groups (housing: $F_{(1,176)}=4.75$, $p=.03, \eta_{\mathrm{p}}^{2}=.03$ ) (Figure $\mathbf{7 B}$, right panel). The USVs subtypes were expressed in percentages relative to the total number of 50$\mathrm{kHz}$ calls. When analyzing the flat USVs within days, the percentage of flats increased from OF1 to OF4, but only in the EE groups (housing: $F_{(3,72)}=2.99, p=.04, \eta_{p}^{2}=.11$; Bonferroni: $p<.05)$ (Figure 7C, left panel). When comparing the housing conditions, EE rats showed a higher call rate than the $\mathrm{SH}$ counterparts, especially in OF3 and OF4 (housing: all $p$-values $\left.<.01, \eta_{\mathrm{p}}^{2}=.16-.17\right)$. A detailed analysis showed that CEE rats had a higher call rate than $\mathrm{SH} 2$ conspecifics on those days (groups: all $p$-values $<.05, \eta_{\mathrm{p}}^{2}=.18-.20$; LSD: $\left.p<.05\right)$. The cumulative percentage of flat calls was higher in the EE groups than in the SH groups (housing: $F_{(1,175)}=17.74, p=.0001, \eta_{p}^{2}=.09$ ), with the CEE rats emitting more flat calls than the SH2 and SH5 counterparts (groups: $F_{(3,173)}=6.76, p=.0001, \eta_{\mathrm{p}}^{2}=.10$ ) (Figure 7C, right panel). Regarding the FM subtypes, the percentage of trills was lower in $\mathrm{EE}$ rats than in $\mathrm{SH}$ counterparts from OF2 onwards (housing: all $p$-values $<.01$, $\eta^{2}{ }_{p}=.14-.18$ ) (Figure 7E, left panel). The single-group analysis revealed that the $\mathrm{CEE}$ rats showed fewer trills than $\mathrm{SH} 2$ counterparts in OF3 and OF4, while the REE rats emitted fewer trills than the SH2 conspecifics only in OF4 (all $p$-values $<.05, \eta_{\mathrm{p}}^{2}=.18-.22$; LSD: $\left.p<.05\right)$. Consequently, the cumulative percentage of trills was lower in both EE groups (housing: $F_{(1,175)}$ $=21.17, p=.0001, \eta_{p}^{2}=.11$ ), with the CEE animals emitting fewer trills than both $\mathrm{SH} 2$ and SH5 animals and the REE rats also emitting fewer calls than the SH2 counterparts (housing: $F_{(1,173)}$ $=8.40, p=.0001, \eta_{p}^{2}=.13$; LSD: $\left.p<.05\right)$ (Figure 7E, right panel). Over days, step-trills reduced from OF1 to OF3 only in EE groups (housing: $F_{(3,72)}=2.77, p=.048, \eta_{p}^{2}=.10$; Bonferroni: $p<$ .05 ) (Figure 7F, right panel). When comparing the housing conditions, a significant and marginal reduction in the call rate was noted on OF3 $\left(p=.03 ; \eta_{p}^{2}=.11\right)$ and OF4 $(p=.08)$, respectively, with no single-group differences detected. For the step-flats, no main effects or interactions were observed (Figure 7E). When comparing the subtype distribution within each group, we found that the $\mathrm{SH} 2$ rats emitted flat calls and trills in almost the same proportion, with both subtypes being higher than the step-flats and step-trills $\left(F_{(3,186)}=50.82, p=.0001, \eta_{\mathrm{p}}^{2}\right.$ $=.45$; Bonferroni: $p<.01$ ) (Figure 7G). In the SH5 groups, the flat calls were 1.1-fold higher than the trills, with both subtypes differing from the rests $\left(F_{(3,111)}=50.04, p=.0001, \eta_{p}^{2}=.58\right.$; Bonferroni: $p<.01)$. In the REE group, the proportion of flat calls was 2.05-fold higher than the trills, with the percentages of these subtypes being higher than that of the step-flats and step-trills $\left(F_{(3,111)}=69.13, p=.0001, \eta_{p}^{2}=.64\right.$; Bonferroni: $\left.p<.01\right)$. In the CEE group, the rate of flat calls was 3.48 -fold higher than the trills, with both call subtypes differing from the rests $\left(F_{(3,108)}=\right.$ 105.27, $p=.0001, \eta_{\mathrm{p}}^{2}=.75$; Bonferroni: $p<.01$ ).

In all groups, the total number of $50-\mathrm{kHz}$ calls reduced during testing minutes but increased throughout OF assessments, with the EE rats showing the lowest rates. This within-test, habituation-like pattern appears after repeated testing and has also been observed in non-EE rats (Wöhr et al., 2008). The lack of differences in call number between the EE groups suggests, again, that partial exposures to $\mathrm{EE}$ are enough to produce the effect of full EE or that motivational factors associated with the REE protocol compensated for the less time they were in the EE cage. In any case, EE may have enhanced the informationprocessing of contextual cues contributing to reducing their social signaling faster than in the $\mathrm{SH}$ groups, when recognizing that no USVs feedback from conspecifics or social encounters would occur within the test. The gradual, between-days increase in call rate observed in all animals may have resulted from a reduction in the averseness of the OF as much as habituation was taking place. Animals tested in mild stress contexts, such as an OF with no bedding, usually display low rates of $50-\mathrm{kHz}$ calls (Wöhr et al., 2008; Natusch and Schwarting, 2010), but when they are repeatedly exposed to familiar (i.e., housing cage with bedding) or unfamiliar (i.e., runway maze) contexts, call rate increases progressively over days (Schwarting et al., 2007; Brenes and Schwarting, 2014). In contrast, if animals are exposed to footshock stress, $50-\mathrm{kHz}$ calls are abolished even when testing occurred in a highly familiar context, such as a housing cage filled with fresh bedding (Rojas-Carvajal and Brenes, submitted). When analyzing the call profile, the reduction in total call number observed in the EE groups coincided with a relative increase in flat calls at the expense of the FM subtypes, especially those including trills. In fact, the percentages of flats and FM calls varied proportionally to the level of social EE, with the rats raised in larger groups emitting more flat calls in the OF, in agreement with previous results (Brenes et al., 2016) (Figure 7G). As separation-induced flat calls may signal a positive affective state of receptiveness to engage in social interactions and to reduce intraspecific aggression (Wöhr et al., 2008; Brenes et al., 2016), increased social contact during early development seems to favor prosocial competence by optimizing the communicational repertoire according to the particular social demands of the context. Also, the differences in the proportion of flat-to-trills percentages observed between the CEE and REE rats suggest USVs are sufficiently responsive to the degree of stimulation that differentiates both EE groups. An alternative, non-exclusive explanation of the differences in the call profile between groups relates to the level of arousal induced by the OF experience. Novelty and exploration are rewarding for rats and 
depend on the activity of the mesolimbic catecholaminergic system (Hughes, 1968; Robinet et al., 1998; for review see Bevins, 2001), with dopamine and especially noradrenaline as the main modulators of the stress and arousal responses (for review, see Godoy et al., 2018). FM calls are also highly dependent on that neurochemical system (for a review, see Simola and Brudzynski, 2018), with noradrenaline activity being critical for psychostimulants to increase FM calls (Wright et al., 2012). Thus, the high percentage of FM USVs seen in the $\mathrm{SH} 2$ rats may reflect a state of arousal induced by the challenging experience of the OF. In the EE rats, on the contrary, the $\mathrm{OF}$ environment was perceived as less novel and exciting in relation to the complexity of the EE cage, which in turn reduced the rate of FM calls. In general, our data showed that the emission of $50-\mathrm{kHz}$ calls was differentially modulated by the degree of EE, both quantitatively and qualitatively.

\section{Experiment 2}

\section{REE Alters Gene Expression in the Hippocampus and Dorsal Striatum}

EE induces numerous physiological, structural, cellular, synaptic, and molecular adaptations, especially in the hippocampus and the mesocorticolimbic circuits (Bekinschtein et al., 2011; Barros et al., 2019; Ohline and Abraham, 2019). Such adaptations comprise changes in signaling pathways and the expression of several genes underlying the neurobehavioral effects of EE (Simpson and Kelly, 2011; Zhang et al., 2018; Ohline and Abraham, 2019). Thus, we measure the mRNA levels of BDNF, TrkB, CREB, DNMT3A, and p250GAP, based on previous studies and because they are integrated within a signaling pathway related with neural plasticity (Nakazawa et al., 2003; Carlezon et al., 2005; Bayraktar and Kreutz, 2018; Kowiański et al., 2018; Sequeira-Cordero et al., 2019)

\section{Hippocampus}

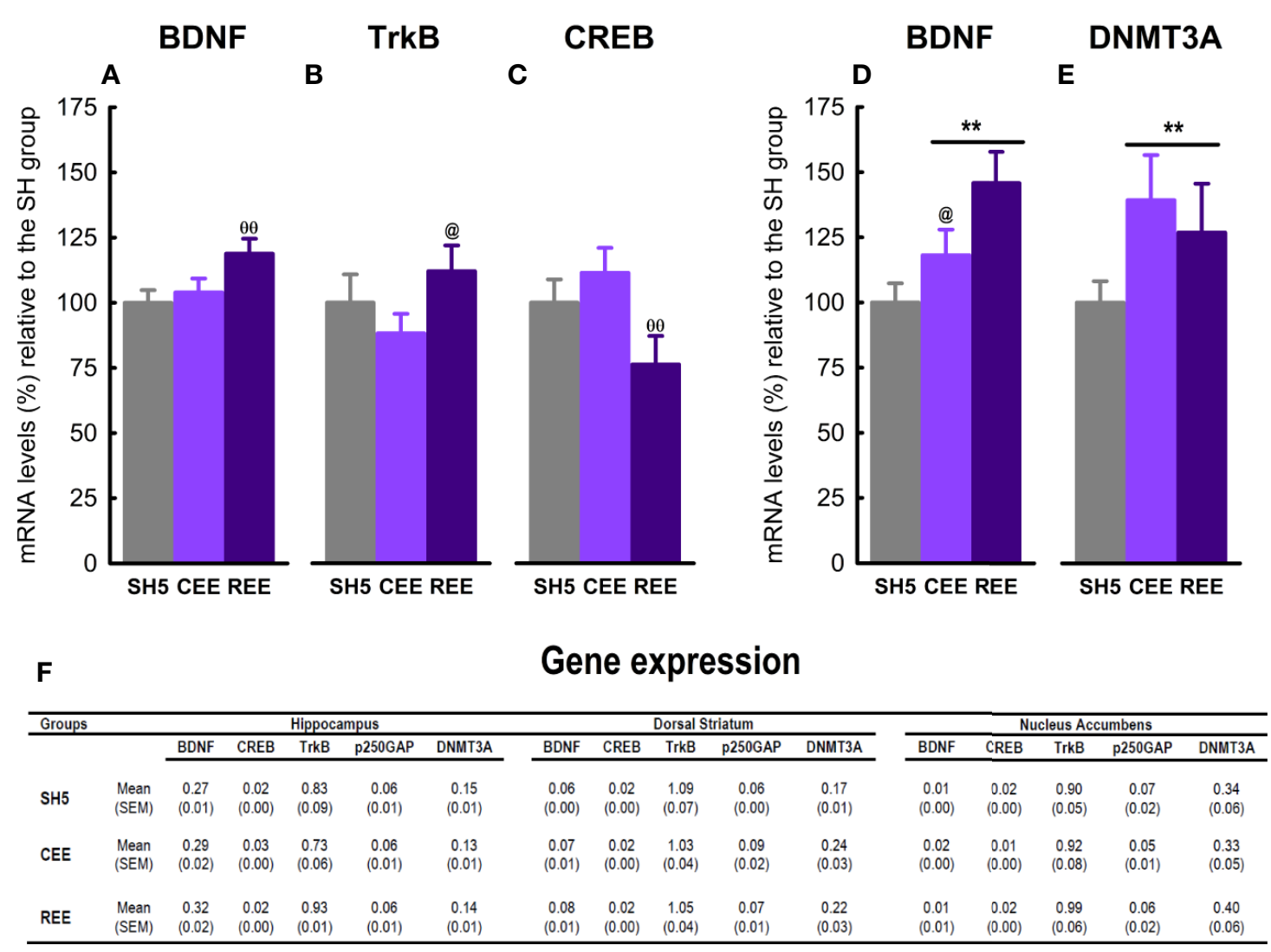

FIGURE 8 | Effects of environmental enrichment on gene expression. Relative mRNA expression of BDNF (A), TrkB (B) and CREB (C) in the hippocampus, and BDNF (D) and DNMT3A (E) in the dorsal striatum. The mRNA expression levels in all the brain regions analyzed (F). Data correspond to percentages relative to the mean of the SH5 group (A-E) or $2^{-\mathrm{dCT}}$ values (F) expressed as mean \pm SEM. SH5, standard housing with five rats. CEE, continuous environmental enrichment; REE, restricted and unpredictable environmental enrichment. Symbols correspond to single, planned comparisons between groups. REE vs. CEE and SH5: ${ }^{\Theta \Theta} p<$ .01. REE vs. CEE: ${ }^{\circledR} p<.05$. CEE and REE vs. SH5: ${ }^{* *} p<.01$. 
(Figure 8F). In a separate experiment, rats were housed in SH5, CEE, and REE conditions ( $n=10$ animals/group) for 30 days and no behavioral testing was performed. After this period, gene expression analyses were carried out on the hippocampus, dorsal striatum, and nucleus accumbens. In the hippocampus, we found that BDNF was upregulated in the REE group as compared to both CEE and SH groups $\left(t_{(27)}=2.60, p=.01, \eta_{\mathrm{p}}^{2}=.21\right)$, which showed very similar levels (Figure 8A). The expression of the TrkB receptor was higher in REE group compared with the CEE group $\left(t_{(27)}=1.78, p=.05, \eta_{p}^{2}=.17\right)$, with no other betweengroup differences detected (Figure 8B). CREB expression was downregulated in the REE group as compared to both CEE and $\mathrm{SH}$ groups $\left(t_{(27)}=2.63, p=.01, \eta_{\mathrm{p}}^{2}=.29\right)$ (Figure 8C). In dorsal striatum, both EE groups showed higher BDNF mRNA levels than the SH group $\left(t_{(27)}=2.63, p=.01, \eta_{p}^{2}=.29\right)$, with the REE group having also higher BDNF expression than CEE group $\left(t_{(27)}\right.$ $=-1.99, p=.05, \eta_{p}^{2}=.24$ ) (Figure 8D). The expression of the DNMT3A was higher in both EE groups compared to the SH group $\left(t_{(26.67)}=2.63, p=.05, \eta_{p}^{2}=.10\right)$ (Figure 8E). No significant differences were observed in the nucleus accumbens.

Experiment 2 aimed to analyze the effects of our EE protocols on the expression of genes involved in neural plasticity and epigenetic regulation in the nucleus accumbens, dorsal striatum, and hippocampus. Although the nucleus accumbens is wellknown to participate in the attribution of incentive salience to reward-predicting cues and appetitive associate learning (Williams and Undieh, 2010; Anselme et al., 2013; Schultz, 2016), no significant differences in gene expression were observed. It is undeniable the nucleus accumbens might have been involved in controlling the motivated responses analyzed in experiment 1, as suggested elsewhere (Burgdorf et al., 2000; Anselme et al., 2013; Schultz, 2016); however, the genes chosen here, the tissue sampling time, and the absence of a behavioral challenge may account for the lack of effects.

Unlike the nucleus accumbens, the hippocampus did show differences in gene expression among groups. For instance, BDNF expression was upregulated only in the REE group, in agreement with one report of intermittent EE (i.e., $12 \mathrm{~h}$ per day throughout 7 weeks) (Williamson et al., 2012). Most of the evidence about the upregulation of hippocampal BDNF mRNA or protein comes from studies of continuous EE in rats (Young et al., 1999; Ickes et al., 2000; Williamson et al., 2012; Bechara and Kelly, 2013) and mice (Rossi et al., 2006; Zajac et al., 2010; Kobilo et al., 2011; Kuzumaki et al., 2011; Chourbaji et al., 2012; Tipyasang et al., 2014; Meng et al., 2015). As we found no differences between SH5 and CEE rats, we failed to reproduce such expected results. Variations in the EE protocols, such as cage size, housing density, type and number of items employed, duration of the protocol, age, and species, may account for the discrepancies (for a review see Simpson and Kelly, 2011). Out of these factors, the inclusion/exclusion of running wheels within the EE cage emerges as a critical methodological aspect, since physical exercise (i.e., the use of running wheels or treadmills) is considered the principal stimulus involved in the EE-induced increases in BDNF expression (Zajac et al., 2010; Kobilo et al., 2011; Bechara and Kelly, 2013). Accordingly, many reports describing higher hippocampal BDNF levels included running wheels in their EE protocol (Young et al., 1999; Ickes et al., 2000; Rossi et al., 2006; Kobilo et al., 2011; Kuzumaki et al., 2011; Williamson et al., 2012; Bechara and Kelly, 2013; Tipyasang et al., 2014; Meng et al., 2015), and at least two studies showing no effects or a decrease in BDNF levels did not use this device (Falkenberg et al., 1992; Spires et al., 2004). Although not definitive, this trend supports the role of exercise in modulating hippocampal BDNF expression and may account for the lack of effects in the CEE group, as we did not include running wheels in our protocol. In REE animals, in contrast, the increased physical activity and incentive motivation may have boosted the stimulatory effects of EE on hippocampal BDNFTrkB expression. Alternatively, as REE animals were enriched only $50 \%$ of the time, the upregulation on those genes might have corresponded to an earlier stage of the EE effects, which may have already returned to baseline levels in the CEE group. Nevertheless, the lack of running wheels did not impede finding differences in BDNF expression in the dorsal striatum, pointing out to a region-dependent effect of our EE protocols on neural plasticity. In that region, BDNF expression was upregulated in both EE groups, with the REE group also showing higher levels than the CEE group, indicating that the dorsal striatum is a very responsive region for identifying activity-dependent changes in the brain. Some studies of continuous EE in mice have found similar (Bezard et al., 2003; Tipyasang et al., 2014) or negative results (Spires et al., 2004; Mazzocchi-Jones et al., 2011). Altogether, our findings suggest that, as compared with $\mathrm{CEE}$, REE recruited the BDNF-TrkB signaling to a greater and broader extent. Although brain and behavioral data corresponded to independent experiments, changes in that signaling pathway may have underlain the behavioral improvements seen in the REE group, as it has been shown with protocols of continuous EE (Bekinschtein et al., 2011; Chourbaji et al., 2011; for a review see Barros et al., 2019).

On the other hand, hippocampal CREB expression was lower only in REE rats than in the other groups, with no further differences observed. To our knowledge, there is no evidence of CREB activity after restricted EE. However, the lack of effects in the CEE group disagrees with many reports showing that continuous EE increases CREB $\mathrm{mRNA}$ /protein or its phosphorylation in the hippocampus of mice (Huang et al., 2006; Huang et al., 2007; Hu et al., 2013; Du et al., 2017) and rats (Young et al., 1999; Zhang et al., 2016). As one report without running wheels failed to reproduce the EE-induced increases in CREB, this factor could explain the discrepancies (Williams et al., 2001). It must also be considered that many studies showing increases in CREB expression included behavioral testing after the EE protocol (Huang et al., 2006; Huang et al., 2007; Zhang et al., 2016; Du et al., 2017). The exposure to novel contexts, such as testing apparatuses, is known to increase CREB expression and phosphorylation (Moncada 
and Viola, 2006). As no behavioral testing was included in experiment 2, the interaction between novelty and EE could have been necessary for upregulating the CREB expression in the hippocampus of CEE rats. A similar interaction between behavioral testing and EE has also been reported for hippocampal BDNF expression (Falkenberg et al., 1992). A third alternative explanation should be considered. Biphasic activation of hippocampal CREB has been related to memory formation, with CREB increasing during novelty encoding and decreasing when the stimulus becomes familiar (Moncada and Viola, 2006). Other reports have also found two peaks of CREB activity following associative learning in rats, suggesting that CREB may follow many activational phases (Bernabeu et al., 1997; Stanciu et al., 2001). In our experiment, it is plausible that the putative increase in CREB expression expected for CEE rats had already occurred at an earlier stage and returned to the baseline levels once the EE cage became familiar. In REE rats, this process may have delayed, first because rats remained in the cage only half of the time and second, due to the constant changes and unpredictable access to the cage, which in turn compromised the familiarization to the context. Thus, the reduction in CREB expression seen in the REE group may have corresponded to that stage. As this panorama remains speculative, further research is needed to clarify the functional relevance of these results.

EE increased the expression of DNMT3A in the dorsal striatum. Although the role of DNMT3A as an epigenetic modifier of EE effects has been scarcely studied, at least one report shows an EE-mediated increase of DNMT3A mRNA in the hippocampus of a genetic mouse model of Alzheimer disease (Griñán-Ferré et al., 2018). Furthermore, it is known that EE modulates gene transcription by increasing or decreasing DNA methylation patterns in a locus-specific manner and depending on the brain region (Griñan-Ferré et al., 2016; Zhang et al., 2018). Thus, our results support the point of view that EE, irrespective of being continuous or random, is capable of augmenting the expression of dorsal striatal DNMT3A, which would have resulted in the hypermethylation and transcriptional regulation of particular loci. Presumably, such epigenetic regulation may have been triggered by the increased sensorimotor and social activity and might somewhat explain its plastic neural adaptations, including the upregulation of BDNF signaling in that region. However, given that such epigenetic changes could operate at myriads of genomic locations, additional studies are required to elucidate the physiologic significance of these results.

\section{DISCUSSION}

In this study, we investigated the likely role of motivation in modulating the neurobehavioral effects of EE in rats. To this aim, we modified our EE protocol by including natural stimuli, which were previously screened and classified according to the speciesspecific behaviors they may elicit. To our knowledge, this is one of the first reports analyzing behavioral kinetics within the EE cage over different time points for 30 days. Also, this is one of the first implementations of a naturalistic EE protocol, and one of the few efforts for describing the protocol in detail, which is a seldom practice in the field leading to replication problems and many inconsistencies within and between labs. Another source of discrepancies is the type of control group used, which varies from social isolation to groups of five or six rats per cage. Here we showed that groups of two or five rats not only differed one another in some parameters but also affected the magnitude at which the significant differences are estimated.

The second and most important implementation of our experiments was the access to the $\mathrm{EE}$ cage, which was made restricted and unpredictable in one EE group. We found that the combination of those factors modified the level and type of activities displayed in the EE cage. Although all EE rats were very active and emitted high rates of $50-\mathrm{kHz}$ calls once entering the cage, the large differences between REE and CEE suggest that the rewarding properties of $\mathrm{EE}$ were perceived as more pronounced in the REE rats. As physical activity and social interaction decline with time (Salchner et al., 2004; for review, see Seffer et al., 2014), intermittent access to the EE stimuli and cagemates may have prolonged the period in which these activities peak. In consequence, the benefits derived from physical and social stimulation could have extended during a developmental window that is very sensitive to EE (for review, see Simpson and Kelly, 2011). Thus, the increased activity and incentive motivation associated with the EE may account for the neurobehavioral effects of the REE, which equaled or even surpassed those observed in the CEE group, although REE rats were only $50 \%$ of the time on EE. We cannot rule out, nevertheless, that for some neurobehavioral parameters, a short EE experience was enough to induce a ceiling effect in which longer EE exposures exerted no further changes. Although the behavioral and brain data were collected on different experiments, it is possible to think that the boost in the BDNF/ TrkB signaling induced by REE was responsible for its behavioral performance. Alternatively, it should be considered that each EE protocol produced its effects following different kinetics, especially at the level of gene expression. As CEE rats accumulated in the first two weeks all the stimulation that REE rats received during one month, the effects seen in the REE brains may have been a rightward shift in the peaks of up- or downregulation in gene expression with their respective behavioral consequences. In the CEE rats, in contrast, all these neurobehavioral dynamics may have diminished or already returned to baseline levels earlier than in REE rats. Further research is warranted to test all these hypotheses experimentally.

Taken together, our results suggest that short but highly rewarding interactions with a stimulating context induce positive effects on brain and behavior, which are comparable or even superior to those produced by a longer period. People living in urban areas spent most of their time working indoors and living in very impoverished environments with little physical and recreational activities during the day (Biernat et al., 2010; Thorp et al., 2012), constituting a lifestyle that has gradually become the "standard housing" of human beings. The reduction of green areas and sports parks in modern cities has jeopardized 
the engagement on recreational activities and physical exercise (Haaland and van Den Bosch, 2015). At best, some subjects will spend few hours a week on exercise or outdoor activities, with most people having occasional and minimal access to "enriching" environments. In worst-case scenarios where these activities are rather limited and social contact could be even prohibited -as is the case of sanitary emergencies caused by viral outbreaks (e.g., AH1N1, SARS-CoV-2)- the opportunities to "get enriched" could be virtually nil. Therefore, the use of short and restricted access to EE and exercise would serve to model many aspects of modern life and may have greater translational value than protocols of continuous exposure to stimulation, especially because it is almost impossible for humans. In this line, providing restricted but highly attractive interventions including physical and social activities, may cause a significant impact on an individual's health at lower time and economic costs and with higher treatment's adherence and efficacy. Thus, the design of personalized interventions based on individual interests and motivations may be a powerful tool for improving or replacing traditional treatments and interventions, which may function as a sort of adjuvant, endogenous pharmacotherapy for patients (Sale et al., 2014). At a different level, the implementation of more attractive educational and recreational programs taking into account personal needs and expectations would benefit disadvantaged infantile populations with limited access to stimulating socio-educational contexts.

\section{DATA AVAILABILITY STATEMENT}

The datasets generated for this study are available on request to the corresponding author.

\section{REFERENCES}

Anselme, P., Robinson, M. J., and Berridge, K. C. (2013). Reward uncertainty enhances incentive salience attribution as sign-tracking. Behav. Brain Res. 238, 53-61. doi: 10.1016/j.bbr.2012.10.006

Ashokan, A., Hegde, A., and Mitra, R. (2016). Short-term environmental enrichment is sufficient to counter stress-induced anxiety and associated structural and molecular plasticity in basolateral amygdala. Psychoneuroendocrinology 69, 189-196. doi: 10.1016/j.psyneuen.2016.04.009

Barros, W., David, M., Souza, A., Silva, M., and Matos, R. (2019). Can the effects of environmental enrichment modulate BDNF expression in hippocampal plasticity? A systematic review of animal studies. Synapse 73 (8), e22103. doi: 10.1002/syn.22103

Bayraktar, G., and Kreutz, M. R. (2018). Neuronal DNA methyltransferases: epigenetic mediators between synaptic activity and gene expression? Neuroscientist 24 (2), 171-185. doi: 10.1177/1073858417707457

Bechara, R. G., and Kelly, A. M. (2013). Exercise improves object recognition memory and induces BDNF expression and cell proliferation in cognitively enriched rats. Behav. Brain Res. 245, 96-100. doi: 10.1016/j.bbr.2013.02.018

Bekinschtein, P., Oomen, C. A., Saksida, L. M., and Bussey, T. J. (2011). Effects of environmental enrichment and voluntary exercise on neurogenesis, learning and memory, and pattern separation: BDNF as a critical variable? Semin. Cell. Dev. Biol. 22 (5), 536-542. doi: 10.1016/j.semcdb.2011.07.002

Benedetti, F., Colloca, L., Torre, E., Lanotte, M., Melcarne, A., Pesare, M., et al. (2004). Placebo-responsive Parkinson patients show decreased activity in single neurons of subthalamic nucleus. Nat. Neurosci. 7 (6), 587-588. doi: 10.1038/nn1250

\section{ETHICS STATEMENT}

The animal study was reviewed and approved by Institutional Committee for Animal Care and Use of the University of Costa Rica (approval number: CICUA-161-16).

\section{AUTHOR CONTRIBUTIONS}

JB, MR-C, and AS-C conceived and designed the experiments. $\mathrm{MR}-\mathrm{C}$ and JB conducted the experiments. MR-C and JB designed and contributed to the analysis of the cage kinetics and the grooming classification. MR-C and JB analyzed the raw behavioral data. AS-C performed the gene expression analysis. MR-C and JB did the statistical analyses. All authors wrote and reviewed the manuscript.

\section{FUNDING}

This research was supported by the projects 837-B7-603 (Neuroscience Research Center), 723-B7-610 (Institute for Psychological Research), and 742-B4-240 (Institute for Health Research), University of Costa Rica. These projects were awarded with the Research Stimulus Fund of the Vice-rectory of Research, University of Costa Rica. We especially thanks to Mónica Sánchez, Brenda Mendez, Fernanda Calderón, David Ramírez and Johanna Akerman for their assistance with this project. We also thank PhD Jaime Fornaguera Trías, technicians, and the administrative personal for their collaboration in this work.

Bernabeu, R., Bevilaqua, L., Ardenghi, P., Bromberg, E., Schmitz, P., Bianchin, M. et al. (1997). Involvement of hippocampal cAMP/cAMP-dependent protein kinase signaling pathways in a late memory consolidation phase of aversively motivated learning in rats. Proc. Natl. Acad. Sci. 94 (13), 7041-7046. doi: 10.1073/pnas.94.13.7041

Bernard, P. B., Castano, A. M., Beitzel, C. S., Carlson, V. B., and Benke, T. A. (2015). Behavioral changes following a single episode of early-life seizures support the latent development of an autistic phenotype. Epil. Beha. 44, 78-85. doi: 10.1016/j.yebeh.2015.01.006

Bevins, R. A. (2001). Novelty Seeking and Reward: Implications for the Study of High-Risk Behaviors. Curr. Direc. Psych. Sci. 10 (6), 189-193. doi: 10.1111/ 1467-8721.00146

Bezard, E., Dovero, S., Belin, D., Duconger, S., Jackson-Lewis, V., Przedborski, S., et al. (2003). Enriched environment confers resistance to 1-methyl-4-phenyl1,2,3,6-tetrahydropyridine and cocaine: involvement of dopamine transporter and trophic factors. J. Neurosc. 23 (35), 10999-11007. doi: 10.1523/ JNEUROSCI.23-35-10999.2003

Biernat, E., Tomaszewski, P., and Milde, K. (2010). Physical activity of office workers. Bio. Sport. 27 (4), 289-296. doi: 10.5604/20831862.927495.

Bonefeld, B. E., Elfving, B., and Wegener, G. (2008). Reference genes for normalization: a study of rat brain tissue. Synapse 62 (4), 302-309. doi: 10.1002/syn.20496

Brenes, J. C., and Fornaguera, J. (2008). Effects of environmental enrichment and social isolation on sucrose consumption and preference: associations with depressive-like behavior and ventral striatum dopamine. Neurosci. Lett. 436 (2), 278-282. doi: 10.1016/j.neulet.2008.03.045 
Brenes, J. C., and Schwarting, R. K. (2014). Attribution and expression of incentive salience are differentially signaled by ultrasonic vocalizations in rats. PloS One 9 (7), e102414. doi: 10.1371/journal.pone.0102414

Brenes, J. C., and Schwarting, R. K. (2015). Individual differences in anticipatory activity to food rewards predict cue-induced appetitive $50-\mathrm{kHz}$ calls in rats. Phys. Behav. 149, 107-118. doi: 10.1016/j.physbeh.2015.05.012

Brenes, J. C., Rodríguez, O., and Fornaguera, J. (2008). Differential effect of environment enrichment and social isolation on depressive-like behavior, spontaneous activity and serotonin and norepinephrine concentration in prefrontal cortex and ventral striatum. Pharma. Biochem. Behav. 89 (1), 8593. doi: 10.1016/j.pbb.2007.11.004

Brenes, J. C., Padilla, M., and Fornaguera, J. (2009). A detailed analysis of openfield habituation and behavioral and neurochemical antidepressant-like effects in postweaning enriched rats. Behav. Brain Res. 197 (1), 125-137. doi: 10.1016/ j.bbr.2008.08.014

Brenes, J. C., Lackinger, M., Höglinger, G. U., Schratt, G., Schwarting, R. K., and Wöhr, M. (2016). Differential effects of social and physical environmental enrichment on brain plasticity, cognition, and ultrasonic communication in rats. J. Comp. Neurol. 524 (8), 1586-1607. doi: 10.1002/cne.23842

Brenes Sáenz, J. C., Rodríguez, O., and Fornaguera, J. (2006). Factor analysis of forced swimming test, sucrose preference test and open field test on enriched, social and isolated reared rats. Behav. Brain Res. 169 (1), 57-65. doi: 10.1016/j.bbr.2005.12.001

Brudzynski, S. M. (2013). Ethotransmission: communication of emotional states through ultrasonic vocalization in rats. Curr. Opin. Neurobiol. 23, 310-317. doi: 10.1016/j.conb.2013.01.014

Burgdorf, J., Knutson, B., and Panksepp, J. (2000). Anticipation of rewarding electrical brain stimulation evokes ultrasonic vocalization in rats. Behav. Neurosc. 114 (2), 320-327. doi: 10.1037/0735-7044.114.2.320

Burgdorf, J., Wood, P. L., Kroes, R. A., Moskal, J. R., and Panksepp, J. (2007). Neurobiology of $50-\mathrm{kHz}$ ultrasonic vocalizations in rats: electrode mapping, lesion, and pharmacology studies. Behav. Brain Res. 182 (2), 274-283. doi: 10.1016/j.bbr.2007.03.010

Burgdorf, J., Kroes, R. A., Moskal, J. R., Pfaus, J. G., Brudzynski, S. M., and Panksepp, J. (2008). Ultrasonic vocalizations of rats (Rattus norvegicus) during mating, play, and aggression: Behavioral concomitants, relationship to reward, and self-administration of playback. J. Comp. Psych. 122 (4), 357-367. doi: $10.1037 / \mathrm{a} 0012889$

Burgdorf, J., Panksepp, J., and Moskal, J. R. (2011). Frequency-modulated 50 kHz ultrasonic vocalizations: a tool for uncovering the molecular substrates of positive affect. Neurosc. Biobehav. Rev. 35 (9), 1831-1836. doi: 10.1016/ j.neubiorev.2010.11.011

Carlezon, W. A.Jr., Duman, R. S., and Nestler, E. J. (2005). The many faces of CREB. Trends Neurosc. 28 (8), 436-445. doi: 10.1016/j.tins.2005.06.005

Chapman, J. R., and Waldenström, J. (2015). With Reference to Reference Genes: A Systematic Review of Endogenous Controls in Gene Expression Studies. PloS One 10 (11), e0141853. doi: 10.1371/journal.pone.0141853

Chourbaji, S., Brandwein, C., and Gass, P. (2011). Altering BDNF expression by genetics and/or environment: impact for emotional and depression-like behaviour in laboratory mice. Neurosci. Biobehav. Rev. 35 (3), 599-611. doi: 10.1016/j.neubiorev.2010.07.003

Chourbaji, S., Hörtnagl, H., Molteni, R., Riva, M. A., Gass, P., and Hellweg, R. (2012). The impact of environmental enrichment on sex-specific neurochemical circuitries - effects on brain-derived neurotrophic factor and the serotonergic system. Neurosc 220, 267-276. doi: 10.1016/ j.neuroscience.2012.06.016

Costa, R., Tamascia, M. L., Nogueira, M. D., Casarini, D. E., and Marcondes, F. K. (2012). Handling of adolescent rats improves learning and memory and decreases anxiety. J. Am. Assoc. Lab. Anim. Sci. 51 (5), 548-553.

Crofton, E. J., Zhang, Y., and Green, T. A. (2015). Inoculation stress hypothesis of environmental enrichment. Neurosc. Biobehav. Rev. 49, 19-31. doi: 10.1016/ j.neubiorev.2014.11.017

Du, L. L., Wang, L., Yang, X. F., Wang, P., Li, X. H., Chai, D. M., et al. (2017). Transient receptor potential-canonical 1 is essential for environmental enrichment-induced cognitive enhancement and neurogenesis. Mol. Neurobio. 54 (3), 1992-2002. doi: 10.1007/s12035-016-9758-9

Ebal, E., Cavalie, H., Michaux, O., and Lac, G. (2007). Effect of a moderate exercise on the regulatory hormones of food intake in rats. Appetite 49 (2), 521-524. doi: 10.1016/j.appet.2007.03.007
Elliott, B. M., and Grunberg, N. E. (2005). Effects of social and physical enrichment on open field activity differ in male and female Sprague-Dawley rats. Behav. Brain Res. 165 (2), 187-196. doi: 10.1016/j.bbr.2005.06.025

Estanislau, C., Veloso, A. W., Filgueiras, G. B., Maio, T. P., Dal-Cól, M. L., Cunha, D. C., et al. (2019). Rat self-grooming and its relationships with anxiety, dearousal and perseveration: Evidence for a self-grooming trait. Phys. Behav. 209, 112585. doi: 10.1016/j.physbeh.2019.112585

Falkenberg, T., Mohammed, A. K., Henriksson, B., Persson, H., Winblad, B., and Lindefors, N. (1992). Increased expression of brain-derived neurotrophic factor mRNA in rat hippocampus is associated with improved spatial memory and enriched environment. Neurosc. Lett. 138 (1), 153-156. doi: 10.1016/0304-3940(92)90494-R

Godoy, L. D., Rossignoli, M. T., Delfino-Pereira, P., Garcia-Cairasco, N., and de Lima Umeoka, E. H. (2018). A Comprehensive Overview on Stress Neurobiology: Basic Concepts and Clinical Implications. Front. Behav. Neurosc. 12, 127. doi: 10.3389/fnbeh.2018.00127

Griñán-Ferré, C., Izquierdo, V., Otero, E., Puigoriol-Illamola, D., Corpas, R., Sanfeliu, C., et al. (2018). Environmental enrichment improves cognitive deficits, $\mathrm{AD}$ hallmarks and epigenetic alterations presented in $5 \mathrm{xFAD}$ mouse model. Front. Cell. Neurosc. 12, 224. doi: 10.3389/fncel.2018.00224

Griñan-Ferré, C., Puigoriol-Illamola, D., Palomera-Ávalos, V., Pérez-Cáceres, D., Companys-Alemany, J., Camins, A., et al. (2016). Environmental enrichment modified epigenetic mechanisms in SAMP8 mouse hippocampus by reducing oxidative stress and inflammaging and achieving neuroprotection. Front. Aging Neurosc. 8, 241. doi: 10.3389/fnagi.2016.00241

Grimm, J. W., Glueck, E., Ginder, D., Hyde, J., North, K., and Jiganti, K. (2018). Sucrose Abstinence and Environmental Enrichment Effects on Mesocorticolimbic DARPP32 in Rats. Sci. Rep. 8 (1), 13174. doi: 10.1038/ s41598-018-29625-X

Haaland, C., and van Den Bosch, C. K. (2015). Challenges and strategies for urban green-space planning in cities undergoing densification: A review. Urb. For. Urb. Green. 14 (4), 760-771. doi: 10.1016/j.ufug.2015.07.009

Hajheidari, S., Miladi-Gorji, H., and Bigdeli, I. (2017). Environmental enrichment prevents methamphetamine-induced spatial memory deficits and obsessivecompulsive behavior in rats. Iran. J. Psych. 12 (1), 8-14.

Hellemans, J., Mortier, G., De Paepe, A., Speleman, F., and Vandesompele, J. (2007). qBase relative quantification framework and software for management and automated analysis of real-time quantitative PCR data. Gen. Bio. 8 (2), R19. doi: 10.1186/gb-2007-8-2-r19

Hendriksen, H., Prins, J., Olivier, B., and Oosting, R. S. (2010). Environmental enrichment induces behavioral recovery and enhanced hippocampal cell proliferation in an antidepressant-resistant animal model for PTSD. PloS One 5 (8), e11943. doi: 10.1371/journal.pone.0011943

Heyse, N., Brenes, J. C., and Schwarting, R. K. W. (2015). Exercise reward induces appetitive $50-\mathrm{kHz}$ calls in rats. Phys. Behav. 147, 131-140. doi: 10.1016/ j.physbeh.2015.04.021

Hirsjärvi, P., and Väliaho, T. (1995). Effects of gentling on open-field behaviour of Wistar rats in fear-evoking test situation. Lab. Anim. 29 (4), 380-384. doi: 10.1258/002367795780739953

Hu, Y. S., Long, N., Pigino, G., Brady, S. T., and Lazarov, O. (2013). Molecular mechanisms of environmental enrichment: impairments in Akt/GSK3 $\beta$, neurotrophin-3 and CREB signaling. PloS One 8 (5), e64460. doi: 10.1371/ journal.pone. 0064460

Huang, F. L., Huang, K. P., and Boucheron, C. (2006). Environmental enrichment enhances neurogranin expression and hippocampal learning and memory but fails to rescue the impairments of neurogranin null mutant mice. J. Neurosci. 26 (23), 6230-6237. doi: 10.1523/JNEUROSCI.1182-06.2006

Huang, F. L., Huang, K. P., and Boucheron, C. (2007). Long-term enrichment enhances the cognitive behavior of the aging neurogranin null mice without affecting their hippocampal LTP. Learn. Mem. 14 (8), 512-519. doi: 10.1101/ $\operatorname{lm} .636107$

Hughes, R. N., and Collins, M. A. (2010). Enhanced habituation and decreased anxiety by environmental enrichment and possible attenuation of these effects by chronic $\alpha$-tocopherol (vitamin E) in aging male and female rats. Pharm. Biochem. Behav. 94 (4), 534-542. doi: 10.1016/j.pbb.2009.11.008

Hughes, R. (1968). Behaviour of male and female rats with free choice of two environments differing in novelty. Anim. Behav. 16, 92-96. doi: 10.1016/00033472(68)90116-4 
Ickes, B. R., Pham, T. M., Sanders, L. A., Albeck, D. S., Mohammed, A. H., and Granholm, A. C. (2000). Long-term environmental enrichment leads to regional increases in neurotrophin levels in rat brain. Exp. Neurol. 164 (1), 45-52. doi: 10.1006/exnr.2000.7415

Julian, G. S., de Oliveira, R. W., Perry, J. C., Tufik, S., and Chagas, J. R. (2014). Validation of Housekeeping Genes in the Brains of Rats Submitted to Chronic Intermittent Hypoxia, a Sleep Apnea Model. PloS One 9 (10), e109902. doi: 10.1371/journal.pone.0109902

Kalueff, A. V., Stewart, A. M., Song, C., Berridge, K. C., Graybiel, A. M., and Fentress, J. C. (2016). Neurobiology of rodent self-grooming and its value for translational neuroscience. Nat. Rev. Neurosc. 17 (1), 45-59. doi: 10.1038/ nrn.2015.8

Kawaguchi, M., Scott, K. A., Moran, T. H., and Bi, S. (2005). Dorsomedial hypothalamic corticotropin-releasing factor mediation of exercise-induced anorexia. Am. J. Phys. Reg. Int. Comp. Phys. 288 (6), R1800-R1805. doi: 10.1152/ajpregu.00805.2004

Kempermann, G., Gast, D., and Gage, F. H. (2002). Neuroplasticity in old age: sustained fivefold induction of hippocampal neurogenesis by long-term environmental enrichment. Ann. Neurol. 52 (2), 135-143. doi: 10.1002/ana.10262

Knutson, B., Burgdorf, J., and Panksepp, J. (1998). Anticipation of play elicits highfrequency ultrasonic vocalizations in young rats. J. Compar. Psych. 112, 65-73. doi: 10.1037/0735-7036.112.1.65

Knutson, B., Taylor, J., Kaufman, M., Peterson, R., and Glover, G. (2005). Distributed neural representation of expected value. J. Neurosci. 25, 48064812. doi: 10.1523/JNEUROSCI.0642-05.2005

Kobilo, T., Liu, Q. R., Gandhi, K., Mughal, M., Shaham, Y., and van Praag, H. (2011). Running is the neurogenic and neurotrophic stimulus in environmental enrichment. Learn. Mem. 18 (9), 605-609. doi: 10.1101/ $\operatorname{lm} .2283011$

Koe, A. S., Ashokan, A., and Mitra, R. (2016). Short environmental enrichment in adulthood reverses anxiety and basolateral amygdala hypertrophy induced by maternal separation. Transl. Psychiat. 6 (2), e729-e729. doi: 10.1038/ tp.2015.217

Kowiański, P., Lietzau, G., Czuba, E., Waśkow, M., Steliga, A., and Moryś, J. (2018). BDNF: a key factor with multipotent impact on brain signaling and synaptic plasticity. Cell. Mol. Neurobio. 38 (3), 579-593. doi: 10.1007/s10571017-0510-4

Kreisler, A. D., Mattock, M., and Zorrilla, E. P. (2018). The duration of intermittent access to preferred sucrose-rich food affects binge-like intake, fat accumulation, and fasting glucose in male rats. Appetite 130, 59-69. doi: 10.1016/j.appet.2018.07.025

Kuzumaki, N., Ikegami, D., Tamura, R., Hareyama, N., Imai, S., Narita, M., et al. (2011). Hippocampal epigenetic modification at the brain-derived neurotrophic factor gene induced by an enriched environment. Hipp 21 (2), 127-132. doi: 10.1002/hipo.20775

Lambert, K., Hyer, M., Bardi, M., Rzucidlo, A., Scott, S., Terhune-Cotter, B., et al. (2016). Natural-enriched environments lead to enhanced environmental engagement and altered neurobiological resilience. Neurosc 330, 386-394. doi: 10.1016/j.neuroscience.2016.05.037

Lisman, J., Buzsáki, G., Eichenbaum, H., Nadel, L., Ranganath, C., and Redish, A. D. (2017). Viewpoints: how the hippocampus contributes to memory, navigation and cognition. Nat. Neurosci. 20 (11), 1434-1447. doi: 10.1038/ nn.4661

Liu, X., Liu, W., Jin, Y., Yu, W., Wang, F., and Liu, L. (2010). Effect of gestational and lactational exposure to perfluorooctanesulfonate on calcium-dependent signaling molecules gene expression in rats' hippocampus. Arch. Toxic. 84 (1), 71-79. doi: 10.1007/s00204-009-0467-2

Marler, K. J., Suetterlin, P., Dopplapudi, A., Rubikaite, A., Adnan, J., Maiorano, N. A, et al.. (2014). BDNF promotes axon branching of retinal ganglion cells via miRNA-132 and p250GAP. J. Neurosc. 34(3), 969-979. doi: 10.1523/ JNEUROSCI.1910-13.2014

Mármol, F., Sánchez, J., Torres, M. N., and Chamizo, V. D. (2017). Environmental enrichment in the absence of wheel running produces beneficial behavioural and anti-oxidative effects in rats. Behav. Process. 144, 66-71. doi: 10.1016/ j.beproc.2017.09.009

Mazzocchi-Jones, D., Döbrössy, M., and Dunnett, S. B. (2011). Environmental enrichment facilitates long-term potentiation in embryonic striatal grafts. Neurorehab. Neural Rep. 25 (6), 548-557. doi: 10.1177/1545968311402090
Meng, F. T., Zhao, J., Ni, R. J., Fang, H., Zhang, L. F., Zhang, Z., et al. (2015). Beneficial effects of enriched environment on behaviors were correlated with decreased estrogen and increased BDNF in the hippocampus of male mice. Neuroendocrino. Let. 36 (5), 490-497.

Mitra, R., and Sapolsky, R. M. (2012). Short-term enrichment makes male rats more attractive, more defensive and alters hypothalamic neurons. PloS One 7 (5), e36092. doi: 10.1371/journal.pone.0036092

Moncada, D., and Viola, H. (2006). Phosphorylation state of CREB in the rat hippocampus: a molecular switch between spatial novelty and spatial familiarity? Neurobio. Learn. Mem. 86 (1), 9-18. doi: 10.1016/ j.nlm.2005.12.002

Mora-Gallegos, A., and Fornaguera, J. (2019). The effects of environmental enrichment and social isolation and their reversion on anxiety and fear conditioning. Behav. Process. 158, 59-69. doi: 10.1016/j.beproc.2018.10.022

Mora-Gallegos, A., Rojas-Carvajal, M., Salas, S., Saborío-Arce, A., FornagueraTrías, J., and Brenes, J. C. (2015). Age-dependent effects of environmental enrichment on spatial memory and neurochemistry. Neurobio. Learn. Mem. 118, 96-104. doi: 10.1016/j.nlm.2014.11.012

Nakazawa, T., Watabe, A. M., Tezuka, T., Yoshida, Y., Yokoyama, K., Umemori, H., et al. (2003). p250GAP, a novel brain-enriched GTPase-activating protein for Rho family GTPases, is involved in the N-methyl-d-aspartate receptor signaling. Mol. Bio. Cell. 14 (7), 2921-2934. doi: 10.1091/mbc.e02-09-0623

Natusch, C., and Schwarting, R. K. W. (2010). Using bedding in a test environment critically affects $50-\mathrm{kHz}$ ultrasonic vocalizations in laboratory rats. Pharm. Biochem. . Behav. 96 (3), 251-259. doi: 10.1016/j.pbb.2010.05.013

Ohline, S. M., and Abraham, W. C. (2019). Environmental enrichment effects on synaptic and cellular physiology of hippocampal neurons. Neuropharm 145, 312. doi: 10.1016/j.neuropharm.2018.04.007

Olsson, I. A., and Dahlborn, K. (2002). Improving housing conditions for laboratory mice: a review of environmental enrichment. Lab. Anim. 36, 243270. doi: 10.1258/002367702320162379

Paumier, K. L., Rizzo, S. J. S., Berger, Z., Chen, Y., Gonzales, C., Kaftan, E., et al. (2013). Behavioral characterization of A53T mice reveals early and late stage deficits related to Parkinson's disease. PloS One 8 (8), e70274. doi: 10.1371/ journal.pone.0070274

Pereira, M., Andreatini, R., Schwarting, R. K., and Brenes, J. C. (2014). Amphetamine-induced appetitive $50-\mathrm{kHz}$ calls in rats: a genuine affective marker of mania? Psychopharm 231, 2567-2577. doi: 10.1007/s00213-0133413-1

Rage, F., Silhol, M., Binamé, F., Arancibia, S., and Tapia-Arancibia, L. (2007). Effect of aging on the expression of BDNF and TrkB isoforms in rat pituitary. Neurobio. Aging. 28 (7), 1088-1098. doi: 10.1016/j.neurobiolaging.2006.05.013

Raymaekers, M., Smets, R., Maes, B., and Cartuyvels, R. (2009). Checklist for optimization and validation of real-time PCR assays. J. Clin. Lab. Analysis. 23 (3), 145-151. doi: 10.1002/jcla.20307

Richard, J. M., Castro, D. C., Difeliceantonio, A. G., Robinson, M. J., and Berridge, K. C. (2013). Mapping brain circuits of reward and motivation: in the footsteps of Ann Kelley. Neurosci. Biobehav. Rev. 37 (9 Pt A), 1919-1931. doi: 10.1016/ j.neubiorev.2012.12.008

Robinet, P. M., Rowlett, J. K., and Bardo, M. T. (1998). Individual differences in novelty-induced activity and the rewarding effects of novelty and amphetamine in rats. Behav. Process. 44 (1), 1-9. doi: 10.1016/S0376-6357(98)00022-9

Rojas-Carvajal, M., Fornaguera, J., Mora-Gallegos, A., and Brenes, J. C. (2018). Testing experience and environmental enrichment potentiated open-field habituation and grooming behaviour in rats. Anim. Behav. 137, 225-235. doi: 10.1016/j.anbehav.2018.01.018

Rojas-Carvajal, M., Brenes, J. C., and Sequeira-Cordero, A. (2019). Age-dependent differences on neurochemistry and behavior in rats raised with low and high levels of maternal care. Behav. Brain. Res. 372, 112054. doi: 10.1016/ j.bbr.2019.112054

Rosenzweig, M. R., and Bennett, E. L. (1996). Psychobiology of plasticity: effects of training and experience on brain and behavior. Behav. Brain. Res. 78 (1), 5765. doi: 10.1016/0166-4328(95)00216-2

Rossi, C., Angelucci, A., Costantin, L., Braschi, C., Mazzantini, M., Babbini, F., et al. (2006). Brain-derived neurotrophic factor (BDNF) is required for the enhancement of hippocampal neurogenesis following environmental enrichment. Europ. J. Neurosc. 24 (7), 1850-1856. doi: 10.1111/j.14609568.2006.05059.x 
Salchner, P., Lubec, G., and Singewald, N. (2004). Decreased social interaction in aged rats may notreflect changes in anxiety-related behaviour. Behav. Brain. Res. 151, 1-8. doi: 10.1016/j.bbr.2003.07.002

Sale, A., Berardi, N., and Maffei, L. (2014). Environment and brain plasticity: towards an endogenous pharmacotherapy. Phys. Rev. 94 (1), 189-234. doi: 10.1152/physrev.00036.2012

Sampedro-Piquero, P., Zancada-Menendez, C., Begega, A., Rubio, S., and Arias, J. L. (2013). Effects of environmental enrichment on anxiety responses, spatial memory and cytochrome c oxidase activity in adult rats. Brain. Res. Bull. 98, 19. doi: 10.1016/j.brainresbull.2013.06.006

Sampedro-Piquero, P., Álvarez-Suárez, P., Moreno-Fernández, R. D., GarcíaCastro, G., Cuesta, M., and Begega, A. (2018). Environmental Enrichment Results in Both Brain Connectivity Efficiency and Selective Improvement in Different Behavioral Tasks. Neurosci 388, 374-383. doi: 10.1016/ j.neuroscience.2018.07.036

Santos, A. R., and Duarte, C. B. (2008). Validation of internal control genes for expression studies: effects of the neurotrophin BDNF on hippocampal neurons. J. Neurosci. Res. 86 (16), 3684-3692. doi: 10.1002/jnr.21796

Scala, F., Nenov, M. N., Crofton, E. J., Singh, A. K., Folorunso, O., Zhang, Y., et al. (2018). Environmental Enrichment and Social Isolation Mediate Neuroplasticity of Medium Spiny Neurons through the GSK3 Pathway. Cell. Rep. 23 (2), 555-567. doi: 10.1016/j.celrep.2018.03.062

Schmitt, U., and Hiemke, C. (1998). Strain differences in open-field and elevated plus-maze behavior of rats without and with pretest handling. Pharm. Biochem. Behav. 59 (4), 807-811. doi: 10.1016/S0091-3057(97)00502-9

Schultz, W. (2016). Dopamine reward prediction error coding. Dial. Clin. Neurosci. 18 (1), 23.

Schwarting, R. K. W., Jegan, N., and Wöhr, M. (2007). Situational factors, conditions and individual variables which can determine ultrasonic vocalizations in male adult Wistar rats. Behav. Brain Res. 182, 208-222. doi: 10.1016/j.bbr.2007.01.029

Scott, D. J., Stohler, C. S., Egnatuk, C. M., Wang, H., Koeppe, R. A., and Zubieta, J. K. (2007). Individual differences in reward responding explain placeboinduced expectations and effects. Neuron 1955 (2), 325-336. doi: 10.1016/ j.neuron.2007.06.028

Seffer, D., Schwarting, R. K. W., and Wöhr, M. (2014). Pro-social ultrasonic communication in rats: Insights from playback studies. J. Neurosci. Meth. 234, 73-81. doi: 10.1016/j.jneumeth.2014.01.023

Sequeira-Cordero, A., Masís-Calvo, M., Mora-Gallegos, A., and Fornaguera-Trías, J. (2013). Maternal behavior as an early modulator of neurobehavioral offspring responses by Sprague-Dawley rats. Behav. Brain. Res. 237, 63-70. doi: 10.1016/j.bbr.2012.09.028

Sequeira-Cordero, A., Salas-Bastos, A., Fornaguera, J., and Brenes, J. C. (2019). Behavioural characterisation of chronic unpredictable stress based on ethologically relevant paradigms in rats. Scient. Rep. 9 (1), 1-21. doi: 10.1038/s41598-019-53624-1

Simola, N., and Brudzynski, S. M. (2018). Rat 50-kHz ultrasonic vocalizations as a tool in studying neurochemical mechanisms that regulate positive emotional states. J. Neurosci. Meth. 1 (310), 33-44. doi: 10.1016/j.jneumeth.2018.06.018

Simpson, J., and Kelly, J. P. (2011). The impact of environmental enrichment in laboratory rats-behavioural and neurochemical aspects. Behav. Brain. Res. 222 (1), 246-264. doi: 10.1016/j.bbr.2011.04.002

Sparling, J. E., Baker, S. L., and Bielajew, C. (2018). Effects of combined pre-and postnatal enrichment on anxiety-like, social, and cognitive behaviours in juvenile and adult rat offspring. Behav. Brain. Res. 353, 40-50. doi: 10.1016/j.bbr.2018.06.033

Spires, T. L., Grote, H. E., Varshney, N. K., Cordery, P. M., Van Dellen, A., Blakemore, C., et al. (2004). Environmental enrichment rescues protein deficits in a mouse model of Huntington's disease, indicating a possible disease mechanism. J. Neurosci. 24 (9), 2270-2276. doi: 10.1523/JNEUROSCI.1658-03.2004

Spruijt, B. M., Van Hooff, J. A., and Gispen, W. H. (1992). Ethology and neurobiology of grooming behavior. Phys. Rev. 72 (3), 825-852. doi: 10.1152/physrev.1992.72.3.825

Stanciu, M., Radulovic, J., and Spiess, J. (2001). Phosphorylated cAMP response element binding protein in the mouse brain after fear conditioning: relationship to Fos production. Mol. Brain. Res. 94 (1-2), 15-24. doi: 10.1016/S0169-328X(01)00174-7

Stein, L. R., O'Dell, K. A., Funatsu, M., Zorumski, C. F., and Izumi, Y. (2016). Short-term environmental enrichment enhances synaptic plasticity in hippocampal slices from aged rats. Neurosci 329, 294-305. doi: 10.1016/ j.neuroscience.2016.05.020

Swaisgood, R. R. (2007). Current status and future directions of applied behavioral research for animal welfare and conservation. Appl. Anim. Behav. Sci. 102 (34), 139-162. doi: 10.1016/j.applanim.2006.05.027

Tarou, L. R., and Bashaw, M. J. (2007). Maximizing the effectiveness of environmental enrichment: Suggestions from the experimental analysis of behavior. Appl. Anim. Behav. Sci. 102 (3), 189-204. doi: 10.1016/ j.applanim.2006.05.026

Tartaglione, A. M., Armida, M., Potenza, R. L., Pezzola, A., Popoli, P., and Calamandrei, G. (2016). Aberrant self-grooming as early marker of motor dysfunction in a rat model of Huntington's disease. Behav. Brain. Res. 313, 53 57. doi: 10.1016/j.bbr.2016.06.058

Thorp, A. A., Healy, G. N., Winkler, E., Clark, B. K., Gardiner, P. A., Owen, N. W., et al. (2012). Prolonged sedentary time and physical activity in workplace and non-work contexts: a cross-sectional study of office, customer service and call center employees. Int. J. Behav. Nutr. Phys. Act. 9 (1), 128. doi: 10.1186/14795868-9-128

Tipyasang, R., Kunwittaya, S., Mukda, S., Kotchabhakdi, N. J., and Kotchabhakdi, N. (2014). Enriched environment attenuates changes in water-maze performance and BDNF level caused by prenatal alcohol exposure. EXCLI. J. 13, 536.

Turner, K. M., and Burne, T. H. (2014). Comprehensive behavioural analysis of Long Evans and Sprague-Dawley rats reveals differential effects of housing conditions on tests relevant to neuropsychiatric disorders. PloS One 9 (3), e93411. doi: 10.1371/journal.pone.0093411

Urakawa, S., Mitsushima, D., Shimozuru, M., Sakuma, Y., and Kondo, Y. (2014). An enriched rearing environment calms adult male rat sexual activity: implication for distinct serotonergic and hormonal responses to females. PloS One 9 (2), e87911. doi: 10.1371/journal.pone.0087911

van Erp, A. M., Kruk, M. R., Meelis, W., and Willekens-Bramer, D. C. (1994). Effect of environmental stressors on time course, variability and form of selfgrooming in the rat: handling, social contact, defeat, novelty, restraint and fur moistening. Behav. Brain. Res. 65 (1), 47-55. doi: 10.1016/0166-4328(94) 90072-8

Van Loo, P. L. P., Van Zutphen, L. F. M., and Baumans, V. (2003). Male management: coping with aggression problems in male laboratory mice. Lab. Anim. 37 (4), 300-313. doi: 10.1258/002367703322389870

Wöhr, M., Houx, B. B., Schwarting, R. K., and Spruijt, B. (2008). Effects of experience and context on $50-\mathrm{kHz}$ vocalizations in rats. Phys. Behav. 93, 766776. doi: 10.1016/j.physbeh.2007.11.031

Widman, D. R., and Rosellini, R. A. (1990). Restricted daily exposure to environmental enrichment increases the diversity of exploration. Phys. Behav. 47 (1), 57-62. doi: 10.1016/0031-9384(90)90042-3

Widman, D. R., Abrahamsen, G. C., and Rosellini, R. A. (1992). Environmental enrichment: the influences of restricted daily exposure and subsequent exposure to uncontrollable stress. Phys. Behav. 51 (2), 309-318. doi: 10.1016/ 0031-9384(92)90146-S

Williams, S. N., and Undieh, A. S. (2010). Brain-derived neurotrophic factor signaling modulates cocaine induction of reward-associated ultrasonic vocalization in rats. J. Pharmacol. Exp. Ther. 332, 463-468. doi: 10.1124/ jpet.109.158535

Williams, B. M., Luo, Y., Ward, C., Redd, K., Gibson, R., Kuczaj, S. A., et al. (2001). Environmental enrichment: effects on spatial memory and hippocampal CREB immunoreactivity. Physiol. Behav. 73 (4), 649-658. doi: 10.1016/S0031-9384 (01)00543-1

Williamson, L. L., Chao, A., and Bilbo, S. D. (2012). Environmental enrichment alters glial antigen expression and neuroimmune function in the adult rat hippocampus. Brain. Behav. Immun. 26 (3), 500-510. doi: 10.1016/ j.bbi.2012.01.003

Wright, J. M., Dobosiewicz, M. R., and Clarke, P. B. (2012). $\alpha$ - and $\beta$-Adrenergic receptors differentially modulate the emission of spontaneous and amphetamine-induced $50-\mathrm{kHz}$ ultrasonic vocalizations in adult rats. Neuropsychopharm 37, 808-821. doi: 10.1038/npp.2011.258

Young, D., Lawlor, P. A., Leone, P., Dragunow, M., and During, M. J. (1999). Environmental enrichment inhibits spontaneous apoptosis, prevents seizures and is neuroprotective. Nat. Med. 5 (4), 448-453. doi: 10.1038/7449

Yu, J. Z., and Rasenick, M. M. (2012). "Receptor signaling and the cell biology of synaptic transmission," in Handbook of Clinical Neurology. Eds. T. E. 
Schlaepfer and C. B. Nemeroff (Amsterdam, The Netherlands: Elsevier), 9-35.

Zajac, M. S., Pang, T. Y. C., Wong, N., Weinrich, B., Leang, L. S. K., Craig, J. M., et al. (2010). Wheel running and environmental enrichment differentially modify exon-specific BDNF expression in the hippocampus of wild-type and pre-motor symptomatic male and female Huntington's disease mice. Hipp 20 (5), 621-636. doi: 10.1002/hipo.20658

Zhang, X. Q., Mu, J. W., Wang, H. B., Jolkkonen, J., Liu, T. T., Xiao, T., et al. (2016). Increased protein expression levels of pCREB, BDNF and SDF-1/ CXCR4 in the hippocampus may be associated with enhanced neurogenesis induced by environmental enrichment. Mol. Med. Rep. 14 (3), 2231-2227. doi: $10.3892 / \mathrm{mmr} .2016 .5470$

Zhang, J., Liu, L. M., and Ni, J. F. (2017). Rapamycin modulated brainderived neurotrophic factor and B-cell lymphoma 2 to mitigate autism spectrum disorder in rats. Neuropsych. Dis. Treat. 13, 835-842. doi: 10.2147/NDT.S125088
Zhang, T. Y., Keown, C. L., Wen, X., Li, J., Vousden, D. A., Anacker, C., et al. (2018). Environmental enrichment increases transcriptional and epigenetic differentiation between mouse dorsal and ventral dentate gyrus. Nat. Commun. 9 (1), 298. doi: 10.1038/s41467-017-02748-x

Conflict of Interest: The authors declare that the research was conducted in the absence of any commercial or financial relationships that could be construed as a potential conflict of interest.

Copyright (๑) 2020 Rojas-Carvajal, Sequeira-Cordero and Brenes. This is an openaccess article distributed under the terms of the Creative Commons Attribution License (CC BY). The use, distribution or reproduction in other forums is permitted, provided the original author(s) and the copyright owner(s) are credited and that the original publication in this journal is cited, in accordance with accepted academic practice. No use, distribution or reproduction is permitted which does not comply with these terms. 